\title{
$71-13,476$
}

KENDALL, James Michael, 1944-

STABILITY DOMAINS IN NONLINEAR POINT

REACTOR DYNAMICS.

University of Arizona, Ph.D., 1971

Engineering, nuclear

University Microfilms, A XEROX Company , Ann Árbor, Michigan 


\section{STABILITY DOMAINS IN NONLINEAR \\ POINT REACTOR DYNAMICS}

by

James Michael Kenda11

A Dissertation Submitted to the Faculty of the DEPARTMENT OF NUCLEAR ENGINEERING

In Partial Fulfillment of the Requirements For the Degree of

DOCTOR OF PHILOSOPHY

In the Graduate College

THE UNIVERSITY OF ARIZONA 
THE UNIVERSITY OF ARIZONA

GRADUATE COLLEGE

I hereby recommend that this dissertation prepared under my direction by JAMES MICHAEL KENDALL

entitled Stability Domains in Nonlinear Point Reactor Dynamics

be accepted as fulfilling the dissertation requirement of the degree of Doctor of Philosophy

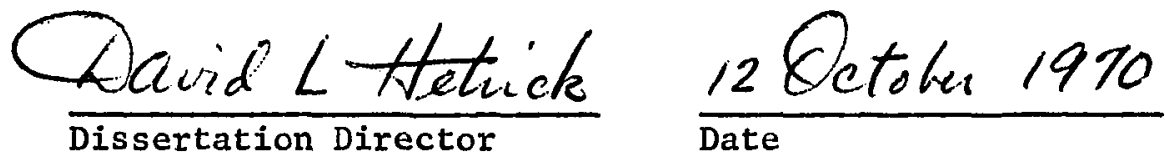

After inspection of the final copy of the dissertation, the following memiers of the Final Examination Committee concur in its approval and recommend its acceptance:*
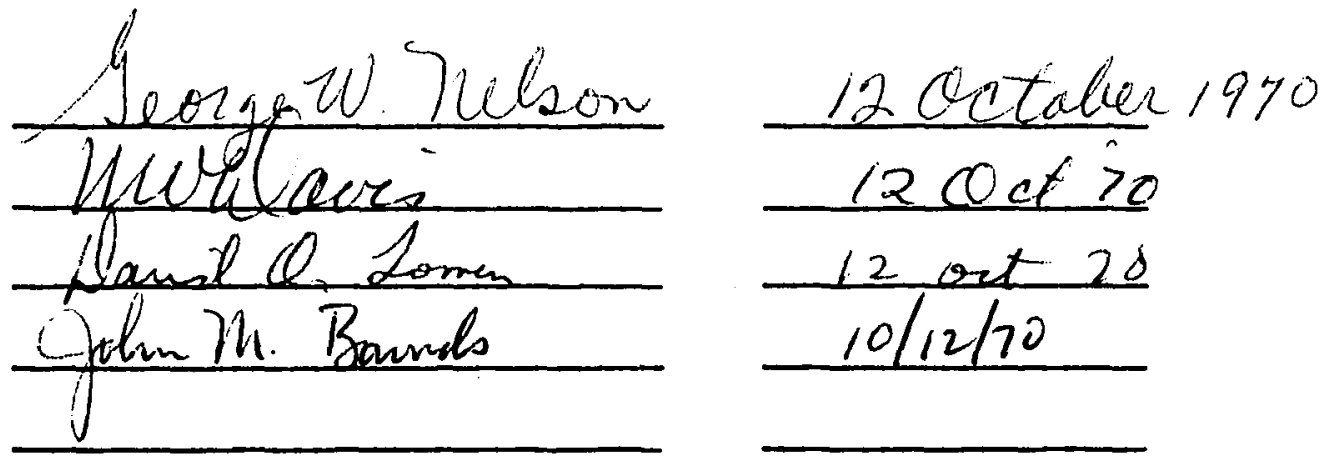

* This approval and acceptance is contingent on the candidate's adequate performance and defense of this dissertation at the final oral examination. The inclusion of this sheet bound into the library copy of the dissertation is evidence of satisfactory performance at the final examination. 


\section{STATEMENT BY AUTHOR}

This dissertation has been submitted in partial fulfillment of requirements for an advanced degree at The University of Arizona and is deposited in the University Library to be made available to borrowers under rules of the Library.

Brief quotations from this dissertation are allowable without special permission, provided that accurate acknowledgment of source is made. Requests for permission for extended quotation from or reproduction of this manuscript in whole or in part may be granted by the head of the major department or the Dean of the Graduate College when in his judgment the proposed use of the material is in the interests of scholarship. In all other instances, however, permission must be obtained from the author.

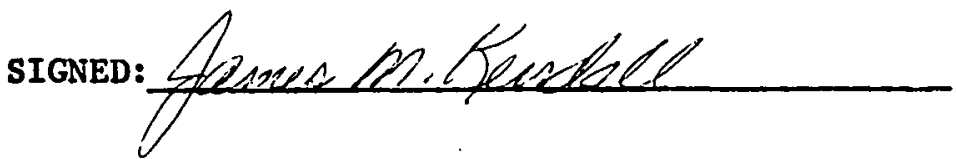




\section{ACKNOWLEDGMENTS}

The author is very grateful to $\mathrm{Dr}$. David L. Hetrick for the advice and direction given throughout the performance of this study.

The author is also indebted to the National Defense Education Act Fellowship Program and the United State Atomic Energy Commission (contract AT-(043)-670) for the financial support which made this work possible. 
TABLE OF CONTENTS

Page

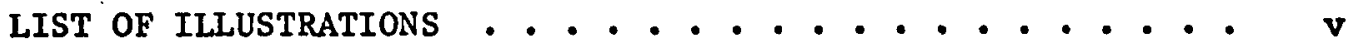

ABSTRACT . . . . . . . . . . . . . . . . vi v

I. INTRODUCTION . . . . . . . . . . . . . . . . . . 1

Point Reactor Kinetics Models . . . . . . . . . . 1

Stability Analysis .................. 4

II. ONE TEMPERATURE REGION WITH POWER COEFFICIENT $\cdot$ - • $\cdot$ - $\cdot$ - 6

Linear Analysis . . . . . . . . . . . . . . 6

Nonlinear Analysis - Exact Solution . . . . . . . . 9

Derivation of Liapunov Function . . . . . . . . . . . 13

Numerical Example ................... 16

III. TWO TEMPERATURE REGIONS - PROMPT POSITIVE FEEDBACK . • • • 22

Linear Analysis . . . . . . . . . . . . . . 23

Nonlinear Analysis - Asymptotic Trajectories . . . . . 26

Nonlinear Analysis - Boundary Planes . . . . . . . . 29

Numerical Example - Decoupled System . . . . . . . . 32

Numerical Example - Coupled System . . . . . . . 33

IV. APPLICATION TO HIGHER ORDER SYSTEMS . . . . . . . . . . 41

One Delay Group, One Region, Power Coefficient .... . 41

Higher Order Systems . . . . . . . . . . . . . . 43

v. SUMMARY . . . . . . . . . . . . . . . . 45

REFERENCES . . . . . . . . . . . . . . . . 47 
1. Parameter Space for One Temperature Region with Power Coefficient Model, $\mathrm{x}=\mathrm{k \gamma} \mathrm{n}_{0} / \ell, \mathrm{y}=\alpha \mathrm{Kn}_{0} / \mathrm{l}$. . . . . 10

2. Comparison of Stable Regions Predicted by Various Methods, One Temperature Region with Power Coefficient Model . . 17

3. System Trajectories, One Temperature Region with Power Coefficient, $\rho_{0}=38$. . . . . . . . . . . . 19

4. System Trajectories, One Temperature Region with Power Coefficient, $\rho_{0}=40$. . . . . . . . . . . . . 20

5. System Trajectories, One Temperature Region with Power Coefficient, $\rho_{0}=42$. . . . . . . . . . . . . . . 21

6. Parameter Space for Two Temperature Region Model, .

$$
x=\alpha_{1} K_{1} n_{0} / l, y=\alpha_{2} K_{2} n_{0} / l, \gamma_{1}>\gamma_{2} \cdots \cdots . . . . . .
$$

7. System Trajectories and Boundary Planes, Two Temperature Region Model, Decoupled System . . . . . . . . . 34

8. Projection of System Trajectories on $n, T$, Plane, Two Temperature Region Model, Decoupled ${ }^{1}$ System . . . . . 35

9. Projection of System Trajectories on $n, T_{2}$ Plane, Two Temperature Region Model, Decoupled ${ }^{2}$ System . . . . . 36

10. Projection of System Trajectories on $T_{1}, T_{2}$ Plane, Two Temperature Region Mode1, Decoupled System . . . . 37

11. Boundary Planes, Two Temperature Region Model,

Coupled System . . . . . . . . . . . . . .

12. Comparison of Boundary Planes to Actual Stability Boundary - 40

13. Projection of System Trajectories on $\mathrm{n}, \mathrm{T}$ Plane, One Delayed Neutron Group, One Region with Power Coefficient . . . 44 


\section{ABSTRACT}

The nonlinear point reactor kinetics model with linear reactivity feedback is studied to determine stable regions of the state space. Geometric and Liapunov techniques are used to determine system stability and effects of system parameters on the stable region.

In studying the one temperature region model with effective lifetime kinetics and a power coefficient, a transformation is obtained which simplifies analysis of the system. Using this transformation an exact solution is found for parameter values such that the system is a nonlinear center. In addition, a Liapunov function is developed which proves asymptotic stability for an infinite sector of the phase space. The stable region shown by this method is compared to those shown by previous techniques for a given set of parameters.

In applying the method to the two temperature region model with effective lifetime kinetics, a set of trajectories asymptotically approaching lines of constant reactivity is shown to exist. An infinite region bounded by three intersecting planes with the property that all trajectories crossing the planes move toward the interior of the region is determined. The parameter condition required for existence of this region is shown to be identical to the condition for the existence of stable limit cycles stated in a previous paper. Numerical examples studied indicate. the region described above is one of bounded trajectories, 1.e., all trajectories with finite initial conditions within the region remain bounded. 
Possible applications of the method to higher order systems is discussed. Preliminary studies indicate a set of trajectories asymptotic to lines of constant reactivity exists for these models, and that this set may be of assistance in an attempt to define regions of stability. 
CHAPTER 1

\section{INTRODUCTION}

In order to ensure safe and reliable operation of a nuclear reactor, its time dependent behavior must be well understood. To obtain exact predictions of the operating characteristics of a reactor, the energy and spatial dependence of the neutron distribution must be included in the calculations. While this can be done by numerical techniques for individual reactor systems, a generalized analytical study including these effects without simplifying assumptions becomes prohibitive if not impossible. For this reason, analytical studies in general have been applied only to highly simplified models. While quantitative predictions of reactor operation using these simplified models should be made with careful attention to the limitations of the model, the results can give qualitative information about effects of varying system parameters. This information can be used to obtain rough estimates of parameter values necessary to achieve desired operational characteristics of the reactor system.

\section{Point Reactor Kinetics Models}

It can be shown (Hetrick, 1971) that by assuming spatial and energy distributions to be Independent of time the source-free Boltzmann transport equation reduces to

$$
\frac{d n}{d t}=\frac{\rho-\beta}{\ell} n+\sum_{i=1}^{m} \lambda_{i} c_{i}
$$




$$
\frac{d c_{i}}{d t}=\frac{\beta_{i}}{\ell} n-\lambda_{i} c_{i} \quad i=1, \ldots, m
$$

where

$$
\begin{aligned}
n(t) & =\text { average neutron density (reactor power) } \\
\rho & =\text { reactivity }=\left(k_{\text {eff }}-1\right) / k_{\text {eff }} \\
\ell & =\text { mean neutron generation time } \\
c_{i}(t) & =\text { average density of the } i^{\text {th }} \text { precursor group } \\
\lambda_{1} & =\text { decay constant of } i^{\text {th }} \text { group } \\
\beta_{1} & =\text { delayed neutron fraction of } i^{\text {th }} \text { group } \\
\beta & =\sum_{i=1}^{m} \beta_{i}=\text { total delayed neutron fraction. }
\end{aligned}
$$

Because of assumptions made in obtaining these equations, they are useful approximations only when the reactor is near critical. For the purposes of this study we will not be concerned with physical restrictions on the system since the work will be directed toward determining characteristics of the mathematical models considered.

The order of the system can be reduced by defining a suitable average delayed neutron precursor decay constant. Although this places further restrictions on the range of validity, the system of equations is reduced to a more manageable form. The most simplified reactor kinetics equation is obtained by neglecting delayed neutron effects entirely or using an adjusted neutron lifetime which reflects their retarding influence on time dependent reactor behavior. In this case the system of equations reduces to 
$\frac{\mathrm{dn}}{\mathrm{dt}}=\frac{\rho}{\ell} \mathbf{n}$.

The differential equation for the average temperature of a region of the reactor is obtained by writing a heat balance assuming heat is refected by Newton cooling. Thus we have

$m_{i} c_{p}^{i} \frac{d_{r}^{i}}{d t}=k_{i} n-u_{i}\left(T_{r}^{i}-T_{c}^{i}\right) i=1, \ldots, q$

where

$$
\begin{aligned}
& m_{i}=\text { mass of material in region } i \\
& c_{p}^{i}=\text { heat capacity } \\
& T_{r}^{i}=\text { temperature } \\
& k_{i}=\text { fraction of reactor power deposited in region } i \\
& u_{i}=\text { overall heat transfer coefficient } \\
& T_{c}^{i}=\text { average coolant temperature. }
\end{aligned}
$$

If we define $T_{i}=T_{r}^{i}-T_{c}^{i}$ and divide by $m_{i} c_{p}^{i}$ we have

$$
\frac{d T_{i}}{d t}=k_{i} n-\gamma_{i} T_{i} 1=1, \ldots, q
$$

For the purposes of this study we assume feedback reactivity is a linear function of $n$ and $T_{i}$, thus

$$
\rho=\rho_{0}-k n-\sum_{i=1}^{q} \alpha_{i} T_{i} \text {. }
$$

Where $\rho_{0}$ is the reactivity of the system at the shutdown point, $\left(n, T_{1}, \ldots T_{q}\right)=(0,0, \ldots 0)$. 
Stability Analysis

System stability as discussed here will be according to the definitions of A. M. Liapunov (Schultz, 1965). For an asymptotically stable region all trajectories with initial conditions within the region approach the equilibrium point as $t$ approaches infinity. Where possible we would like to show this type of stability; however, for higher order systems the trajectories may remain bounded without approaching the equilibrium point. If all trajectories with finite initial conditions within a region remain bounded in time we call this a region of bounded trajectories. An example of this type of behavior is a system with a stable limit cycle and unstable equilibrium point. Obviously the system is not asymptotically stable, yet a region exists with the property that trajectories with initial conditions within the region remain bounded for all time.

In attempting to define properties of the models studied we will use geometric theory and, where possible, Liapunov functions. In using geometric theory we attempt to define surfaces in the phase space with the property that all trajectories cross the surface in a particular direction. In addition, we will seek a set of surfaces which together form a closed surface with the property that all trajectories cross the boundary of the closed surface toward the interior. If this can be done, the region defined will be a region of bounded trajectories.

In this study we will attempt to find planar surfaces with the properties mentioned above. These surfaces can then be used in an attempt to define regions of bounded trajectories. In addition, the planes can be used to define variable transformations which may simplify 
derivation of suitable Liapunov functions. To determine the relation of surfaces obtained to actual stability boundaries for particular parameter values, trajectories are calculated using the GSVDQ subroutine. This subroutine uses predictor-corrector techniques for calculating trafectories from a given set of initial conditions and the system differential equations. 


\section{CHAPTER 2}

- ONE TEMPERATURE REGION WITH POWER COEFFICIENT

The kinetics equations for this model are

$$
\begin{aligned}
& \frac{d n}{d t}=\frac{\rho_{0}-\alpha T-k n}{\ell} n \\
& \frac{d T}{d t}=k n-\gamma T
\end{aligned}
$$

\section{Linear Analysis}

The equilibrium points are

$$
\begin{aligned}
& \text { (1) Shutdown equilibrium: }(n, T)=(0,0) \\
& \text { (2) Operating equilibrium: }(n, T)=\left(n_{0}, T_{0}\right)
\end{aligned}
$$

where $\mathrm{n}_{0}$ and $\mathrm{T}_{0}$ satisfy

$$
\begin{aligned}
& \rho_{0}-\alpha \mathbf{T}_{0}-k \mathbf{n}_{0}=0 \\
& \mathbf{n}_{0}-\gamma \mathrm{T}_{0}=0
\end{aligned}
$$

Linearizing about the shutdown point we let $n=\delta n, T=\delta T$, and neglecting second order terms we have

$$
\begin{aligned}
& \frac{d \delta n}{d t}=\frac{\rho}{l} \delta n \\
& \frac{d \delta T}{d t}=K \delta n-\gamma \delta T
\end{aligned}
$$

solving for the eigenvalues, $\lambda_{1}=\rho_{0} / \ell, \lambda_{2}=-\gamma$. For the parameter values of interest, $\rho_{0} / \ell>0, \gamma>0$, the elgenvalues are real and of opposite sign, thus the shutdown equilibrium is a saddle point. 
Linearizing about the operating equilibrium we let $\mathbf{n}=\mathbf{n}_{0}+\delta \mathbf{n}$, $T=T_{0}+\delta T$, and we have

$$
\begin{aligned}
& \frac{d \delta n}{d t}=\frac{\rho_{0}-\alpha T_{0}-\alpha \delta T-k n_{0}-\kappa \delta n}{\ell}\left(n_{0}+\delta n\right) \\
& \frac{d \delta T}{d t}=K_{0}+k \delta n-\gamma T_{0}-\gamma \delta T
\end{aligned}
$$

rearranging,

$$
\begin{aligned}
& \frac{d \delta n}{d t}=\frac{\rho_{0}^{-\alpha T_{0}}-\kappa n_{0}}{\ell}\left(n_{0}+\delta n\right)-\frac{\alpha \delta T+\kappa \delta n}{\ell}\left(n_{0}+\delta n\right) \\
& \frac{d \delta T}{d t}=\left(K_{0}-\gamma T_{0}\right)+(K \delta n-\gamma \delta T) .
\end{aligned}
$$

From Eqs. (2-3) and (2-4) we see that the first terms of the above equations vanish and dropping second order terms we have

$$
\begin{aligned}
& \frac{d \delta \mathbf{n}}{d t}=-\frac{\alpha n_{0}}{\ell} \delta T-\frac{k n_{0}}{\ell} \delta n \\
& \frac{d \delta T}{d t}=K \delta n-\gamma \delta T .
\end{aligned}
$$

Defining the feedback reactivity as $\rho_{f}=\alpha \delta T+k \delta n$, we can solve for the forward loop transfer function defined by

$$
G(s)=\frac{\delta n(s)}{\rho(s)}=\frac{n_{0}}{l^{s}}
$$

The feedback transfer function is given by

$$
H(s)=\frac{\rho_{f}(s)}{\delta n(s)}=k+\alpha \frac{\delta T(s)}{\delta n(s)}
$$

taking the Laplace transform of Eq. (2-8) we have

$$
s \delta T(s)=K \delta n(s)-\gamma \delta T(s)
$$


or

$$
\frac{\delta T(s)}{\delta n(s)}=\frac{K}{s+\gamma}
$$

Thus the feedback transfer function is

$$
H(s)=k+\frac{\alpha K}{s+\gamma}
$$

We now use the Routh criterion for determining linear stability

with

$$
1+G(s) H(s)=1+\frac{n_{0}}{\ell s}\left(K+\frac{\alpha K}{s+\gamma}\right)
$$

multiplying through by $s(s+\gamma)$ and grouping terms we have

$$
D(s)=s^{2}+\left(\gamma+\frac{k n_{0}}{\ell}\right) s+\frac{\alpha K+K \gamma}{\ell} n_{0}
$$

The Routh array is

$$
\begin{array}{cc}
1 & \frac{\alpha \mathrm{K}+k \gamma}{\ell} \mathrm{n}_{0} \\
\gamma+\frac{k n_{0}}{\ell} & 0 \\
\frac{\alpha \mathrm{K}+k \gamma}{\ell} \mathrm{n}_{0} & 0
\end{array}
$$

The conditions required for linear stability are

$$
\begin{aligned}
& \gamma+\frac{k n_{0}}{\ell}>0 \\
& \frac{\alpha K+k Y}{\ell} n_{0}>0
\end{aligned}
$$

To display these conditions in a parameter space we let $x=k \gamma n_{0} / l, y=a k n_{0} / l$, and the above inequalities become 


$$
\begin{aligned}
& x>-\gamma^{2} \\
& x+y>0
\end{aligned}
$$

The region satisfying these inequalities is shown in Fig. 1. The nonlinear analysis in the following sections will be restricted to parameter values such that $x<0$ (positive power coefficient).

\section{Nonlinear Analysis - Exact Solution}

Studying this system for $\alpha>0, k<0$, it can be easily shown that the constant slope isoclines ( $d n / d T=$ constant) approach a set of straight lines of slope $-\alpha / k$ for $n$ and $T$ large. Thus the isocline $\mathrm{dn} / \mathrm{dT}=-\alpha / \mathrm{k}$ is approached by a solution trajectory for large n and T. Computer solutions to sample problems indicate this trajectory forms the boundary between stable and unstable regions of the state space. If this is true, the linear asymptote for this trajectory could provide information about nonlinear stability of the system. We now assume a solution of the form $n=A T+B$ and solve for the coefficients. In order to have a solution we need $\mathrm{dn} / \mathrm{dt}=\mathrm{AdT} / \mathrm{dt}$, with $\mathbf{n}=\mathrm{AT}+\mathrm{B}$ for $\mathrm{T}$ large.

$$
\begin{aligned}
\frac{d n}{d t} & =\frac{\rho_{0}-\alpha T-K n}{\ell} \mathfrak{n} \\
& =\frac{\rho_{0}^{-\alpha T-K A T-K B}}{\ell}(A T+B) \\
& =\frac{\rho_{0}-K B}{\ell} B+\left(\frac{\rho_{0}-K B}{\ell} A-\frac{\alpha+K A}{\ell} B\right) T-\frac{\alpha+K A}{\ell} A T^{2} \\
A \frac{d T}{d t} & =A K n-A \gamma T \\
& =A K(A T+B)-A \gamma T \\
& =A B K+A(A K-\gamma) T .
\end{aligned}
$$




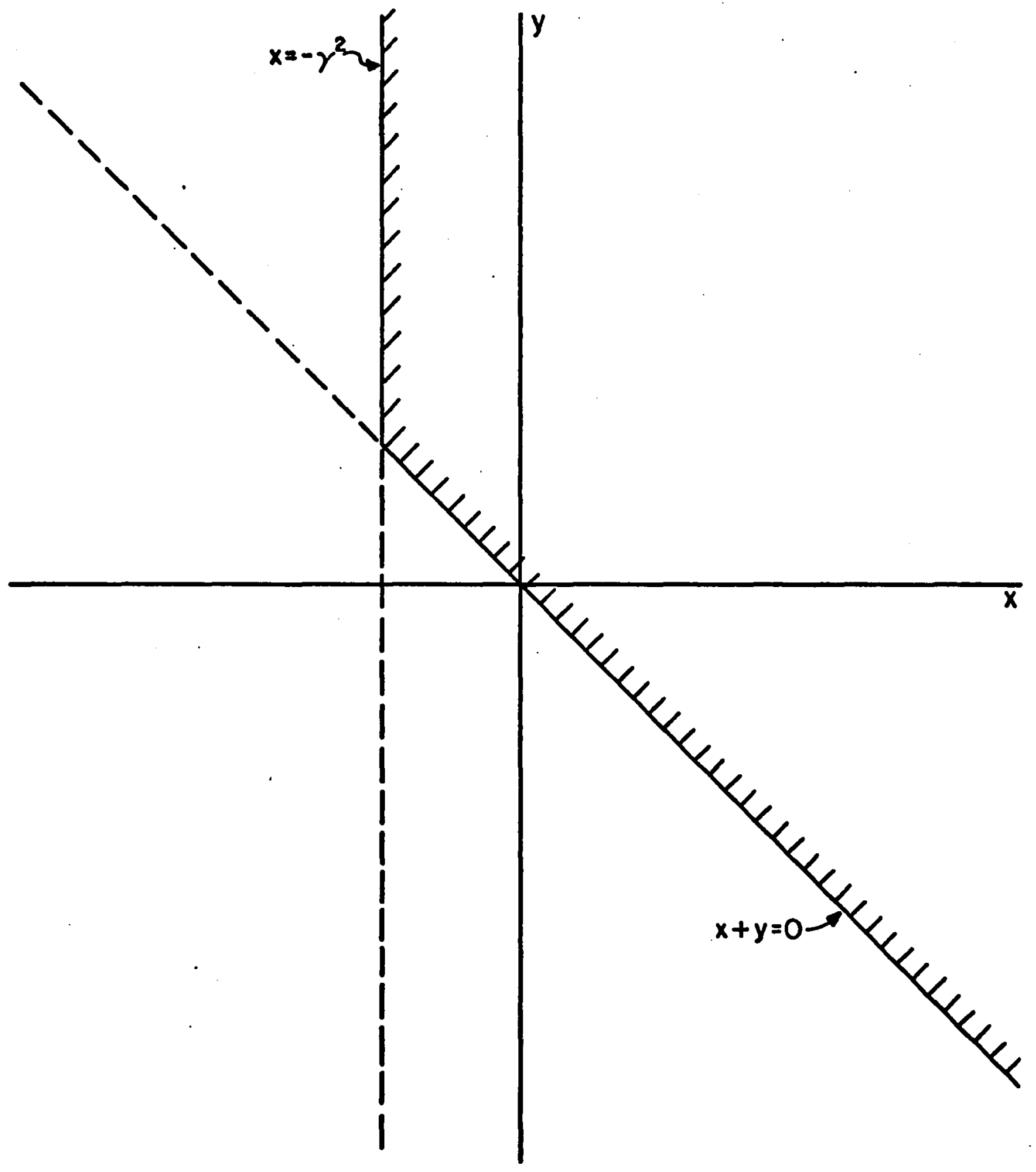

Fig. 1. Parameter Space for One Temperature Region with Power Coefficient Model, $x=k \gamma n_{0} / l, y=\alpha K n_{0} / l$ 
Setting the $\mathrm{T}^{2}$ term to zero we have

$$
\alpha+\kappa A=0 \text {. }
$$

Equating the coefficients of the linear terms we have

$$
\frac{\rho_{0}-K B}{\ell} A=A(A K-\gamma)
$$

Solving for $A$ and $B$,

$$
\begin{aligned}
& A=-\frac{\alpha}{K} \\
& B=\frac{\ell}{K}\left(\frac{\rho_{0}}{\ell}+\gamma+\frac{\alpha K}{K}\right) .
\end{aligned}
$$

Thus the equation for the straight line is

$$
n=-\frac{\alpha}{k} T+\frac{\ell}{k}\left(\frac{\rho}{\ell}+\gamma+\frac{\alpha K}{k}\right)
$$

or,

$$
\rho_{0}-\alpha T-k n+l\left(\gamma+\frac{\alpha K}{k}\right)=0
$$

We now use this insight to make the following transformation:

$$
\begin{aligned}
& \mathbf{u}=\frac{\alpha T+k n-\rho_{0}}{\ell}-\left(\gamma+\frac{\alpha K}{k}\right) \\
& v=-\frac{k}{\ell} n
\end{aligned}
$$

In this space the operating equilibrium becomes

$$
\begin{aligned}
& u_{0}=-\frac{\rho_{0}-\alpha T_{0}-k n_{0}}{\ell}-\left(\gamma+\frac{\alpha K}{K}\right)=-\left(\gamma+\frac{\alpha K}{K}\right) \\
& v_{0}=-\frac{k}{\ell} n_{0}=-\frac{k}{\ell} \frac{\gamma \rho_{0}}{\alpha K+K \gamma}=\frac{\gamma \rho_{0}}{\ell u_{0}}
\end{aligned}
$$

Solving for the differential equations in the transformed system,

$$
\frac{d u}{d t}=\frac{\alpha}{l} \frac{d T}{d t}+\frac{k}{l} \frac{d n}{d t}
$$




$$
\begin{aligned}
& =\frac{\alpha K}{l} \mathbf{n}-\frac{\alpha Y}{\ell} \mathbf{T}+\frac{K}{\ell} \frac{\rho_{\ell}-\alpha \mathbf{T}-\kappa \mathfrak{n}}{\ell} \mathbf{n} \\
& =\frac{\alpha K}{k} \frac{k}{\ell} n+\gamma \frac{k}{\ell} n-\gamma \frac{\alpha T+k n}{\ell}+v\left(u-u_{0}\right) \\
& =-\left(\frac{\alpha K}{K}+\gamma\right) v-\gamma\left(\frac{\rho_{0}}{\ell}+u-u_{0}\right)+v\left(u-u_{0}\right) \\
& =u_{0} v-\frac{\gamma \rho_{0}}{\ell}-\gamma\left(u-u_{0}\right)+u v-u_{0} v \\
& =u v-u_{0} v_{0}-\gamma\left(u-u_{0}\right) \\
& =u v-u v_{0}+u v_{0}-u_{0} v_{0}-\gamma\left(u-u_{0}\right) \\
& \frac{d u}{d t}=u\left(v-v_{0}\right)+\left(v_{0}-\gamma\right)\left(u-u_{0}\right) \\
& \frac{d v}{d t}=-\frac{k}{l} \frac{d n}{d t} \\
& =-\frac{k}{\ell} \frac{\rho_{0}-\alpha T-\kappa \mathfrak{n}}{\ell} \mathfrak{n} \\
& \frac{d v}{d t}=-v\left(u-u_{0}\right)
\end{aligned}
$$

If the parameters are such that $v_{0}=\gamma$, the equations can be integrated directly to obtain trajectories,

$$
\begin{aligned}
& \frac{d v}{d u}=-\frac{v\left(u-u_{0}\right)}{u\left(v-v_{0}\right)} \\
& \left(1-\frac{u_{0}}{u}\right) d u+\left(1-\frac{v_{0}}{v}\right) d v=0
\end{aligned}
$$

Integrating from initial conditions $u_{i}, v_{i}$, we have

$$
\begin{aligned}
& \int_{u_{i}}^{u}\left(1-\frac{u_{0}}{u^{\prime}}\right) d u !^{\prime}+\int_{v_{1}}^{v}\left(1-\frac{v_{0}}{v^{T}}\right) d v^{\prime}=0 \\
& \left(u-u_{1}\right)-u_{0} \ln \frac{u}{u_{1}}+\left(v-v_{1}\right)-v_{0} \ln \frac{v}{v_{1}}=0 .
\end{aligned}
$$


In the region $u>0, v>0$, this is the equation of a set of closed curves; thus the solution is a nonlinear center for parameter values such that $v_{0}=\gamma \cdot$

$$
\begin{aligned}
& \text { Transforming the solution back to } n, T \text { space we have } \\
& \frac{\alpha T+k n-\rho_{0}}{\ell}-\left(\gamma+\frac{\alpha K}{k}\right)-\frac{\alpha T_{i}+k n_{i}-\rho_{0}}{\ell}+\left(\gamma+\frac{\alpha K}{k}\right)+\left(\gamma+\frac{\alpha K}{k}\right) . \\
& \ln \left[\left(\frac{\alpha T+k n-\rho_{0}}{\ell}-\gamma-\frac{\alpha K}{k}\right) /\left(\frac{\alpha T_{i}+k n_{i}-\rho_{0}}{\ell}-\gamma-\frac{\alpha K}{k}\right)\right] \\
& -\frac{k}{l} n+\frac{k}{\ell} n_{i}+\frac{k n_{\theta}}{\ell} \ln \frac{n}{n_{i-}}=0
\end{aligned}
$$

Noting that $v_{0}=\gamma$ implies $\rho_{0} / \ell=-(\alpha K / K+\gamma)$ and cancelling terms we have

$$
\frac{\alpha}{\ell}\left(T-T_{i}\right)-\frac{\rho_{0}}{\ell} \ln \frac{\alpha T+k n}{\alpha T_{i}+k n_{i}}-\gamma \ln \frac{n}{n_{i}}=0 .
$$

This equation forms a set of closed curves in the region $n>0, \alpha T+k n>0$ of $\mathrm{n}, \mathrm{T}$ space. The condition $\mathrm{v}_{0}=\gamma=-\mathrm{x}_{0} / l$ corresponds to Eq. (2-9) of the line bounding linear stability in the parameter space.

\section{Derivation of Liapunov Function}

In seeking a Liapunov function for this system we will apply the variable gradient method (Schultz, 1965), in $\xi_{1} \xi_{2}$ space, where $\xi_{1}=u-u_{0}, \xi_{2}=v-v_{0}$. The differential equations become

$$
\begin{aligned}
& \frac{d \xi_{1}}{d t}=\left(\xi_{1}+u_{0}\right) \xi_{2}+\left(v_{0}-\gamma\right) \xi_{1} \\
& \frac{d \xi_{2}}{d t}=-\left(\xi_{2}+v_{0}\right) \xi_{1}
\end{aligned}
$$


14

We write the gradient of the Liapunov function in the form

$$
\nabla V=\left|\begin{array}{lll}
a_{11} & \xi_{1}+a_{12} & \xi_{2} \\
a_{21} & \xi_{1}+a_{22} & \xi_{2}
\end{array}\right|
$$

Thus,

$$
\begin{aligned}
\frac{d V}{d t} & =\nabla V \cdot \frac{d \xi}{d t} \\
& =\left(a_{11} \xi_{1}+a_{12} \xi_{2}\right)\left[\left(\xi_{1}+u_{0}\right) \xi_{2}+\left(v_{0}-\gamma\right) \xi_{1}\right] \\
& -\left(a_{21} \xi_{1}+a_{22} \xi_{2}\right)\left(\xi_{2}+v_{0}\right) \xi_{1} \\
\frac{d V}{d t} & =\left[a_{11}\left(v_{0}-\gamma\right)-a_{21}\left(\xi_{2}+v_{0}\right)\right] \xi_{1}^{2} \\
& +\left[a_{11}\left(\xi_{1}+u_{0}\right)+a_{12}\left(v_{0}-\gamma\right)-a_{22}\left(\xi_{2}+v_{0}\right)\right] \xi_{1} \xi_{2} \\
& +a_{12}\left(\xi_{1}+u_{0}\right) \xi_{2}^{2}
\end{aligned}
$$

Let

$$
\begin{aligned}
& a_{12}=a_{21}=0 \\
& \frac{d V}{d t}=a_{11}\left(v_{0}-\gamma\right) \xi_{1}^{2}+\left[a_{11}\left(\xi_{1}+u_{0}\right)-a_{22}\left(\xi_{2}+v_{0}\right)\right] \xi_{1} \xi_{2}
\end{aligned}
$$

Setting the $\xi_{1} \xi_{2}$ term to zero,

$$
a_{11}\left(\xi_{1}+u_{0}\right)=a_{22}\left(\xi_{2}+v_{0}\right)
$$

Following the convention used by Schultz we want to restrict $a_{11} a_{22}$ to functions of $\xi_{1}, \xi_{2}$ respectively, let

$$
a_{11}=\frac{i}{\xi_{1}+u_{0}}, a_{22}=\frac{1}{\xi_{2}+v_{0}}
$$


thus we have

$$
\frac{d V}{d t}=\frac{v_{0}-\gamma}{\xi_{1}+u_{0}} \quad \xi_{1}^{2}
$$

For $v_{0}<\gamma$ this function is negative semi-definite in the region $\xi_{1}>-u_{0}$ and it is easily shown that $\xi_{1}=0$ does not contain a semi-trajectory of the system.

$$
\begin{aligned}
& \text { We now derive } V\left(\xi_{1}, \xi_{2}\right) \text { from } \\
& \begin{aligned}
\mathrm{v}\left(\xi_{1}, \xi_{2}\right) & =\int_{0}^{\xi_{1}} \nabla v_{1}\left(\xi_{1}^{\prime}, 0\right) \mathrm{d} \xi_{1}^{\prime}+\int_{0}^{\xi_{2}} \nabla v_{2}\left(\xi_{1}, \xi_{2}^{\prime}\right) \mathrm{d} \xi_{2}^{\prime} \\
& =\int_{0}^{\xi_{1}} \frac{\xi_{1}^{\prime}}{\xi_{1}^{\prime}+u_{0}} \mathrm{~d} \xi_{1}^{\prime}+\int_{0}^{\xi_{2}} \frac{\xi_{2}^{\prime}}{\xi_{2}^{\prime}+v_{0}} \mathrm{~d} \xi_{2}^{\prime}
\end{aligned} \\
& \begin{aligned}
\mathrm{v}\left(\xi_{1}, \xi_{2}\right) & =\xi_{1}-u_{0} \ln \frac{\xi_{1}+u_{0}}{u_{0}}+\xi_{2}-v_{0} \ln \frac{\frac{\xi_{2}}{v_{0}} v_{0}}{v_{0}} .
\end{aligned}
\end{aligned}
$$

This function is positive definite in the region $\xi_{1}>-u_{0}, \xi_{2}>-v_{0}$, and approaches infinity at all boundaries of the region, thus the system is asymptotically stable in the region provided $v_{0}<\gamma$.

Writing the Liapunov function in terms of the $u, v$ variables we have

$$
v(u, v)=u-u_{0}-u_{0} \ln \frac{u}{u_{0}}+v-v_{0}-v_{0} \ln \frac{v}{v_{0}} .
$$

Note that the $V=c o n s t a n t$ curves have the same form as the trajectories of the nonlinear center. In this space the time derivative is

$$
\frac{d V}{d t}=-\frac{\gamma-v_{0}}{u}\left(u-u_{0}\right)^{2}
$$


Transforming back to $n, T$ space the function beçomes

$$
\begin{aligned}
V(n, T) & =\frac{\alpha}{\ell}\left(T-T_{0}\right)+\frac{k n_{0}}{\ell} \ln \frac{n}{n_{0}} \\
& +\left(\gamma+\frac{\alpha K}{k}\right) \operatorname{lil}\left[\left(\frac{\rho_{0}-\alpha T-k n}{\ell}+\gamma+\frac{\alpha K}{k}\right) /\left(\gamma+\frac{\alpha K}{K}\right)\right]
\end{aligned}
$$

the time derivative is

$$
\frac{d V}{d t}=-\frac{\gamma+k n_{0} / l}{\left(\alpha T+k n-\rho_{0}\right) / l-\gamma-\alpha K / k}\left(\rho_{0}-\alpha T-k n\right)^{2}
$$

Asymptotic stability is proven in the region $n>0,(\alpha T+k n) / \ell>\rho_{0} / \ell+\gamma+\alpha K / k$, with the restrictions $k<0, \gamma+\kappa n_{0} / \ell>0, \gamma+\alpha K / \kappa<0$.

\section{Numerical Example}

The following parameters will be used in this section:

$$
\alpha / \ell=1, k / l=-1, K=50, \gamma=10, \rho_{0} / \ell=6 \text {. }
$$

For the purpose of comparing with results of other methods these parameters have been taken from previous papers (Shotkin, 1969; Devooght and Smets, 1967).

For this case the straight line bounding the region of proven asymptotic stability is given by

$$
n=T+34
$$

The Liapunov function is

$$
v(n, T)=(T-7.5)-40 \ln \frac{T-n+34}{40}-1.5 \ln \frac{n}{1.5}
$$

Figure 2 is a comparison of stable regions predicted by various methods for the parameters used here. For this case the stable region 


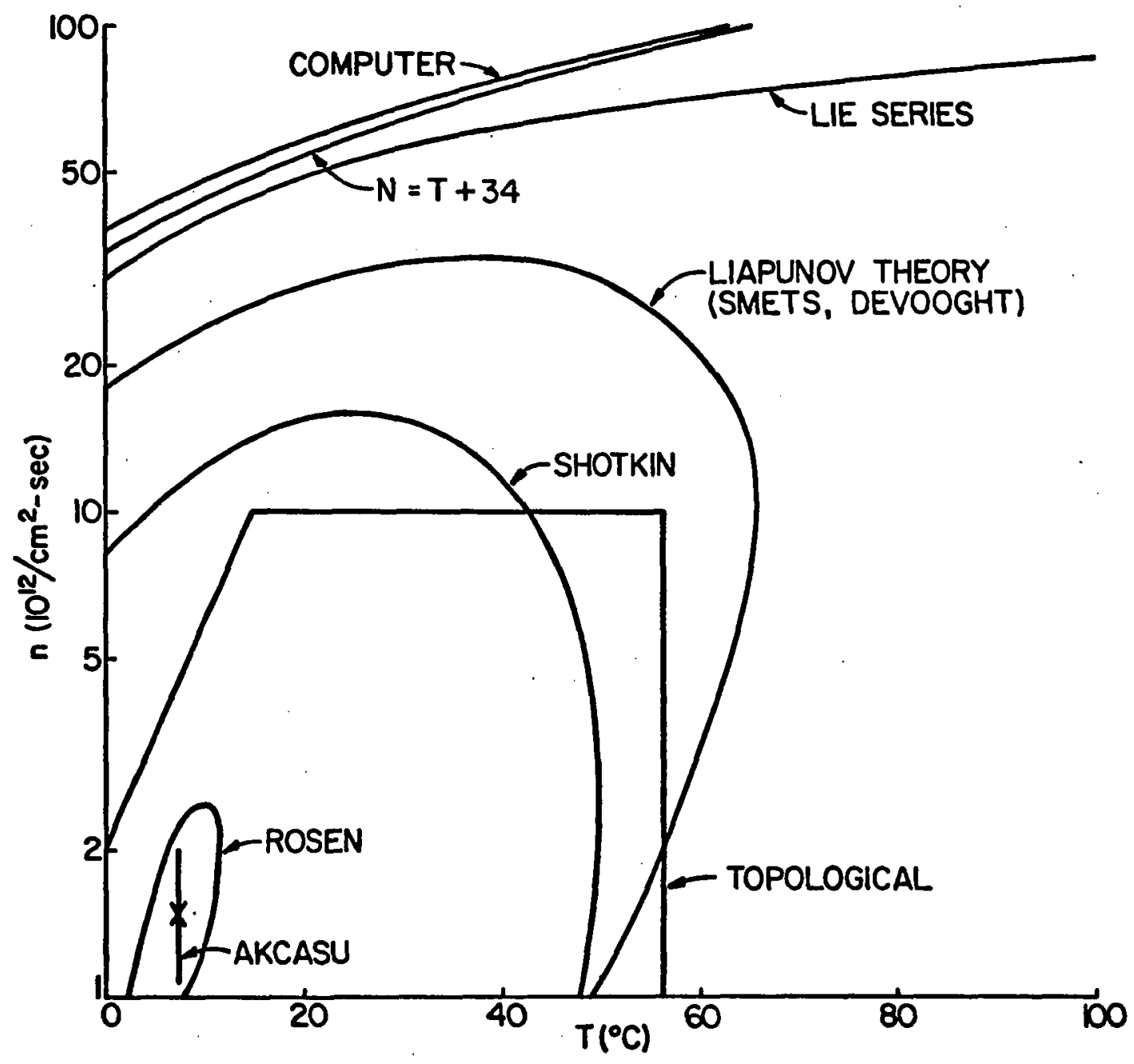

Fig. 2. Comparison of Stable Regions Predicted by Varlous Methods, One Temperature Region with Power Coefficient Model 
demonstrated by the above Liapunov function is much larger than regions shown by previous methods and contains all of these regions except for a small part of the region of Devooght and Smets (for large negative $\mathrm{T}$ ).

For the parameters used here the nonlinear center can be attained by increasing $\rho_{0} / \ell$ to 40 . Figures 3,4 , and 5 show system trajectories for $\rho_{0} / \ell$ less than, equal to and greater than 40. As $n_{0}$ passes through the critical value - $\frac{\gamma l}{K}$ the system changes from asymptotically stable to unstable. 


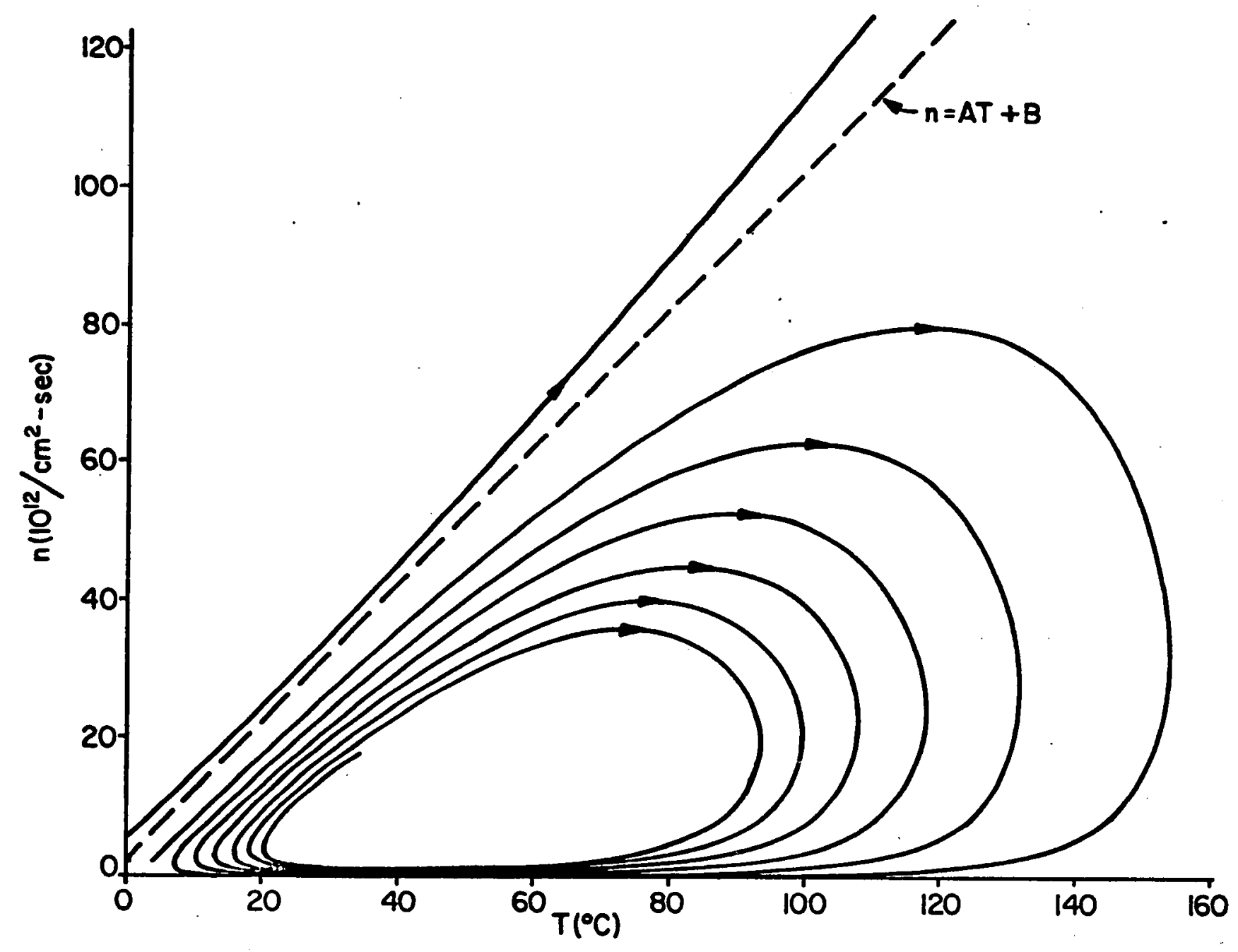

F1g. 3. System Trajectories, One Temperature Region with Power Coefficient, $p_{0}=38$ 


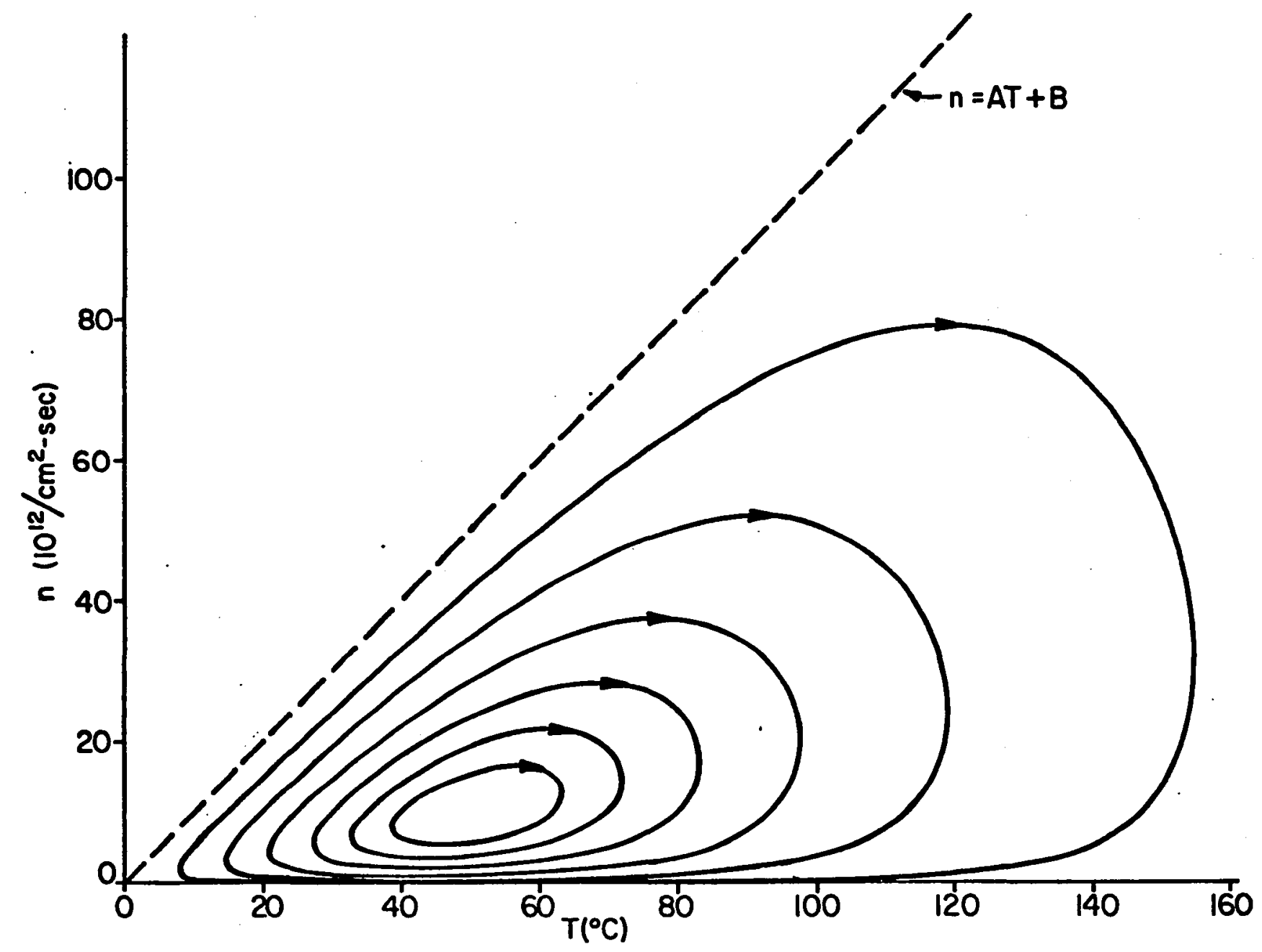

Fig. 4. System Trajectories, One Temperature Region with Power Coefficient, $\rho_{0}=40$ 


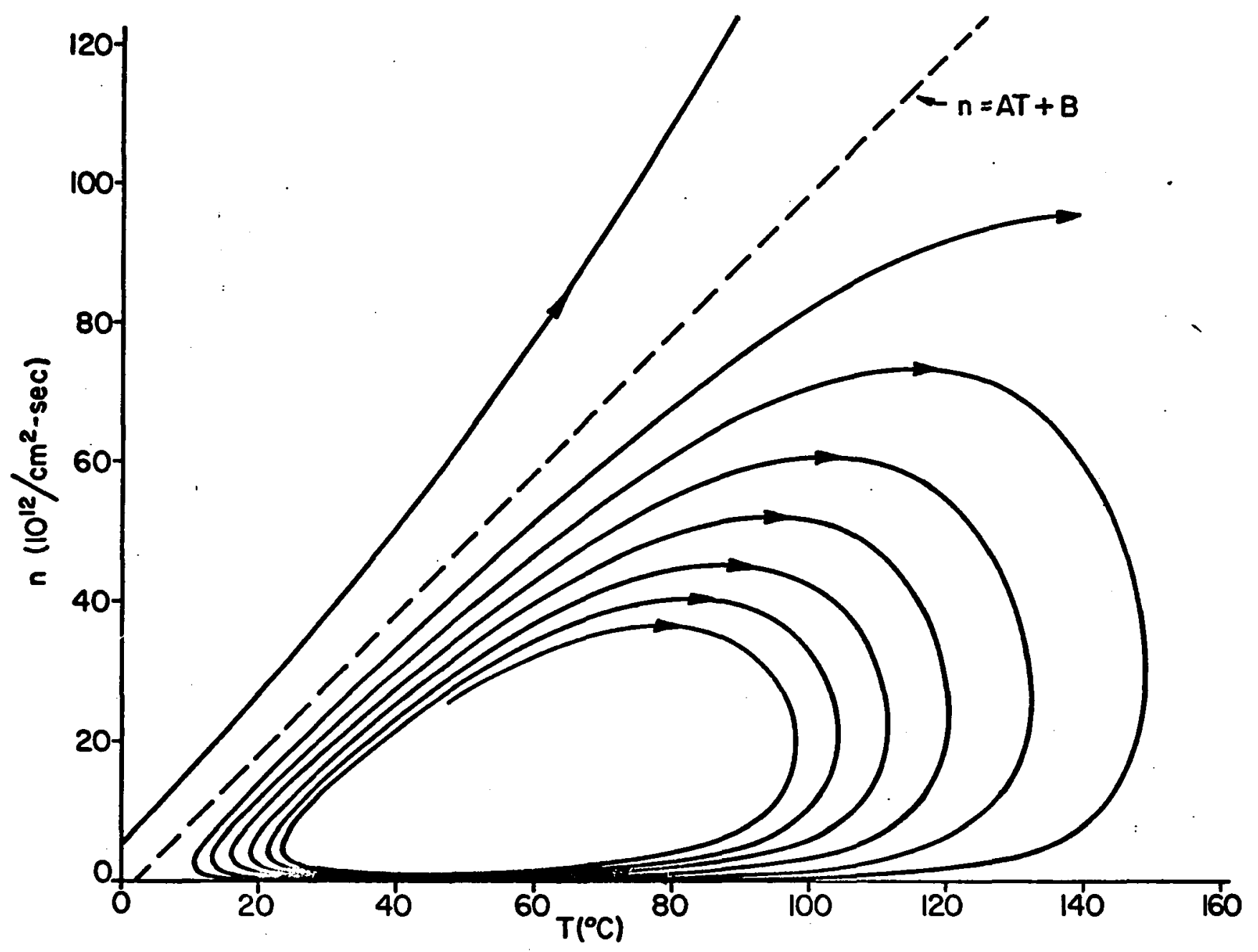

Fig. 5. System Trajectories, One Temperature Region with Power Coefficient, $\rho_{0}=42$ 
CHAPTER 3

TWO TENPERATURE REGIONS - PRONPT POSITIVE FEEDBACK

We now consider the case of a reactor with two regions from which heat is being transferred. Two models will be studied; the first model is for a case where heat is generated and transferred independently from both regions. The differential equations are

$$
\begin{aligned}
& \frac{d n}{d t}=\frac{\rho_{0}-\alpha_{1} T_{1}-\alpha_{2} T_{2}}{\ell} n \\
& \frac{d T_{1}}{d t}=K_{1} n-\gamma_{1} T_{1} \\
& \frac{d T_{2}}{d t}=K_{2} n-\gamma_{2} T_{2}
\end{aligned}
$$

For the second case we consider a system where heat is generated in one region and transferred through a second region. In this model the differential equations are

$$
\begin{aligned}
& \frac{d n}{d t}=\frac{\rho_{0}-\alpha_{1} T_{1}-\alpha_{2} T_{2}}{\ell} n \\
& \frac{d T}{d t}=b n-n_{1} T_{1} \\
& \frac{d T}{d t}=n_{2} T_{1}-n_{3} T_{2} .
\end{aligned}
$$

Later In the chapter these models will be shown to be related by a simple linear transformation. 


\section{Linear Analysis}

Because of the equivalence of the two systems we will only study the linear characteristics of the first model (decoupled system). Setting the time derivatives to zero we obtain equations for the operating equilibrium

$$
\begin{aligned}
& \rho_{0}-\alpha_{1} T_{1}^{0}-\alpha_{2} T_{2}^{0}=0 \\
& K_{1} n_{0}-\gamma_{1} T_{1}^{0}=0 \\
& K_{2} n_{0}-\gamma_{2} T_{2}^{0}=0 .
\end{aligned}
$$

Letting $n=n_{0}+\delta n, T_{1}=T_{1}^{0}+\delta T_{1}, T_{2}=T_{2}^{0}+\delta T_{2}$ and substituting into the differential equations we have

$$
\begin{aligned}
& \frac{d \delta n}{d t}=\frac{\rho_{0}-\alpha_{1} T_{1}^{0}-\alpha_{1} \delta T_{1}-\alpha_{2} T_{2}^{0}-\alpha_{2} \delta T_{2}}{l}\left(n_{0}+\delta n\right) \\
& \frac{d \delta T_{1}}{d t}=K_{1} n_{0}+K_{1} \delta n-\gamma_{1} T_{1}^{0}-\gamma_{1} \delta T_{1} \\
& \frac{d \delta T_{2}}{d t}=K_{2} n_{0}+K_{2} \delta n-\gamma_{2} T_{2}^{0}-\gamma_{2} \delta T_{2}
\end{aligned}
$$

Regrouping terms,

$$
\begin{aligned}
& \frac{d \delta n}{d t}=\frac{\rho_{0}-\alpha_{1} T_{1}^{0}-\alpha_{2} T_{2}^{0}}{l}\left(n_{0}+\delta n\right)-\frac{\alpha_{1} \delta T_{1}+\alpha_{2} \delta T_{2}}{l}\left(n_{0}+\delta n\right) \\
& \frac{d \delta T}{d t}=\left(K_{1} n_{0}-\gamma_{1} T_{1}^{0}\right)+\left(K_{1} \delta n-\gamma_{1} \delta T_{1}\right) \\
& \frac{d \delta T}{d t}=\left(K_{2} n_{0}-\gamma_{2} T_{2}^{0}\right)+\left(K_{2} \delta n-\gamma_{2} \delta T_{2}\right)
\end{aligned}
$$


From the equations satisfied by the equilibrium point we see that the first term is zero in all three cases. Dropping second order terms, the linearized equations are

$$
\begin{aligned}
& \frac{d \delta \mathfrak{n}}{d t}=-\frac{\alpha_{1} n_{0}}{\ell} \delta T_{1}-\frac{\alpha_{2} n_{0}}{l} \delta T_{2} \\
& \frac{d \delta T_{1}}{d t}=K_{1} \delta n-\gamma_{1} \delta T_{1} \\
& \frac{d \delta T_{2}}{d t}=K_{2} \delta n-\gamma_{2} \delta T_{2} .
\end{aligned}
$$

For this case we define the feedback reactivity as $\rho_{f}=\alpha_{1} \delta T$ $+\alpha_{2} \delta T_{2}$, thus the forward loop transfer function is

$$
G(s)=\frac{\delta n(s)}{R(s)}=\frac{n_{0}}{\ell s}
$$

the feedback transfer function is

$$
H(s)=\frac{\rho_{f}(s)}{\delta n(s)}=\alpha_{1} \frac{\delta T_{1}(s)}{\delta n(s)}+\alpha_{2} \frac{\delta T_{2}(s)}{\delta n(s)}
$$

Taking the Laplace transform of the linearized equations, we have

$$
\frac{\delta T_{1}(s)}{\delta n(s)}=\frac{k_{1}}{s+\gamma_{1}}
$$

Thus the feedback transfer function is

$$
H(s)=\frac{\alpha_{1} K_{1}}{s+\gamma_{1}}+\frac{\alpha_{2} K_{2}}{s+\gamma_{2}}
$$

Applying the Routh criterion for this case we have

$$
1+G(s) H(s)=1+\frac{n_{0}}{2 s}\left(\frac{\alpha_{1} K_{1}}{s+\gamma_{1}}+\frac{\alpha_{2} K_{2}}{s+\gamma_{2}}\right)
$$


Multiplying by $s\left(s+\gamma_{1}\right)\left(s+\gamma_{2}\right)$,

$$
\begin{aligned}
D(s) & =s^{3}+\left(\gamma_{1}+\gamma_{2}\right) s^{2}+\left(\gamma_{1} \gamma_{2}+\frac{\alpha_{1} k_{1}+\alpha_{2} k_{2}}{\ell} n_{0}\right) s \\
& +\frac{\alpha_{1} k_{1} \gamma_{2}+\alpha_{2} k_{2} \gamma_{1}}{l} n_{0}
\end{aligned}
$$

Define the following quantities to simplify notation

$$
\begin{aligned}
& k=\frac{\alpha_{1} k_{1}+\alpha_{2} K_{2}}{\ell} n_{0} \\
& \xi=\frac{\alpha_{1} k_{1} \gamma_{2}+\alpha_{2} k_{2} \gamma_{1}}{\alpha_{1} k_{1}+\alpha_{2} k_{2}}
\end{aligned}
$$

We now have

$$
D(s)=s^{3}+\left(\gamma_{1}+\gamma_{2}\right) s^{2}+\left(\gamma_{1} \gamma_{2}+k\right) s+k \xi
$$

The Routh array for this model is

$$
\begin{array}{cc}
1 & \gamma_{1} \gamma_{2}+k \\
\gamma_{1}+\gamma_{2} & K \xi \\
\gamma_{1} \gamma_{2}+k-\frac{K \xi}{\gamma_{1}+\gamma_{2}} & 0 \\
K \xi & 0
\end{array}
$$

For physical reasons we can restrict $\gamma_{1}$ and $\gamma_{2}$ to be positive, and the conditions for linear stability about the operating equilibrium are

$$
\begin{aligned}
& K E_{1}>0 \\
& Y_{1} Y_{2}+K-\frac{K \xi}{Y_{1}+\gamma_{2}}>0
\end{aligned}
$$


Looking at these results in a parameter space we let $x=\alpha_{1} K_{1} n_{0} / \ell \quad y=\alpha_{2} K_{2} n_{0} / l$, and assume without loss of generality that $\gamma_{1}>\gamma_{2}$ (this corresponds to assigning $T_{1}$ to the region with the fastest time response). From the first Routh condition we obtain

$$
\gamma_{2} x+\gamma_{1} y>0
$$

The second condition is given by

$$
\gamma_{1} x+\gamma_{2} y+\gamma_{1} \gamma_{2}\left(\gamma_{1}+\gamma_{2}\right)>0 \text {. }
$$

The line bounding the region satisfying the above inequalities is line DGF on Fig. 6.

Since the equilibrium power $\mathrm{n}_{0}$, which is proportional to the shutdown reactivity $\rho_{0}$, is contained in both parameters, the effect of changing $\rho_{0}$ is to move along a straight line which passes through the origin. If the reactor parameters are such that we are in region $A O E$, the shutdown reactivity can be increased to a point where the system becomes linearly unstable. In this region the system remains linearly stable under the condition that the equilibrium power be held below a certain level (conditional stability).

\section{Nonlinear Analysis - Asymptotic Trajectories}

Based on the results obtained in the previous chapter, it is reasonable to expect that some insight can be gained for the two region model through the same technique. Since we are now working in a three dimensional space, two linear equations are needed to define a line in the space. Assume these are of the form $\mathrm{n}=A \mathrm{~T}_{2}+\mathrm{B}$, and $\mathrm{T}_{1}=\mathrm{CT}_{2}+\mathrm{D}$. 
27

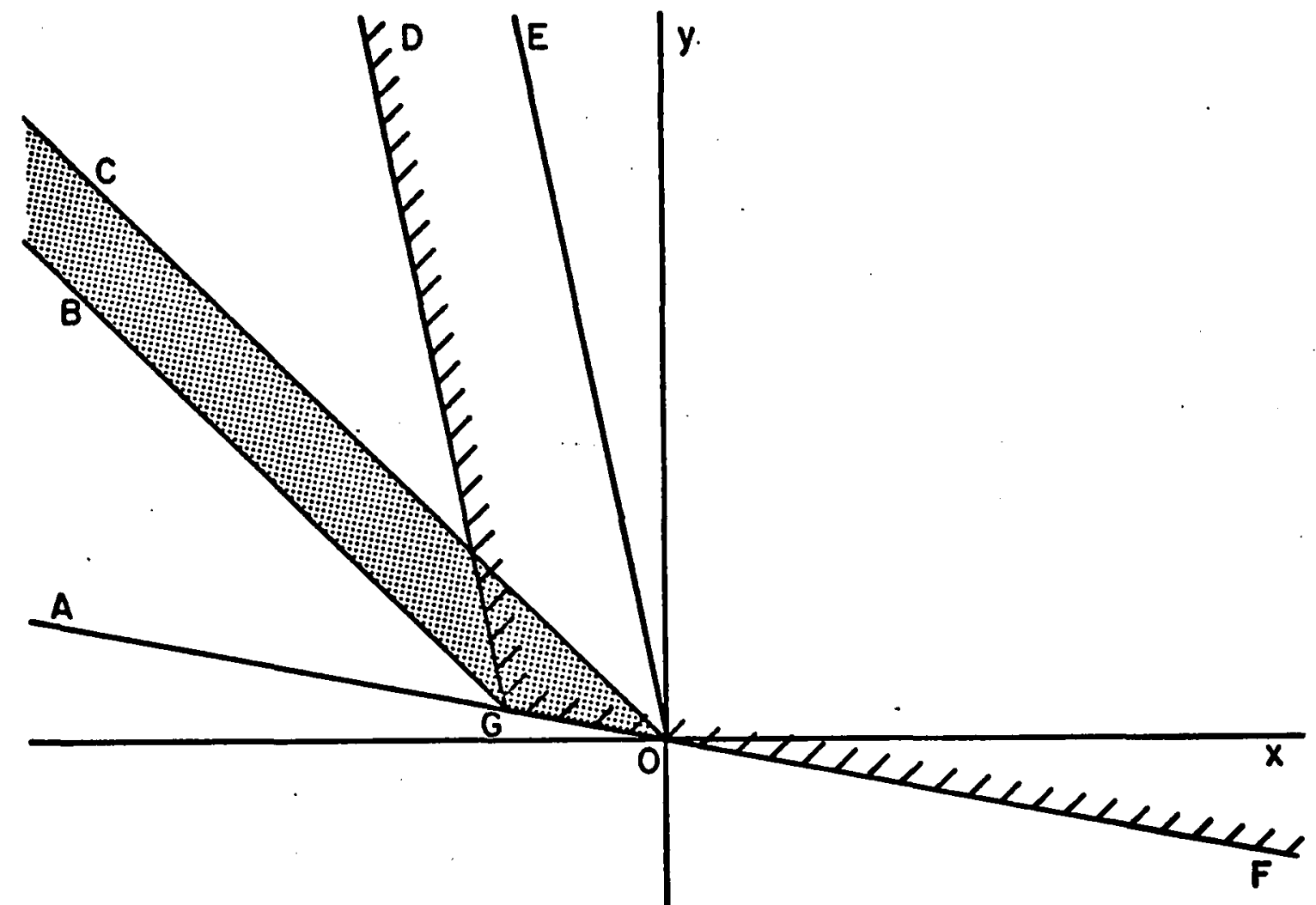

Fig. 6. Parameter Space for Two Temperature Region Model, $x=\alpha_{1} k_{1} n_{0} / 2, y=\alpha_{2} k_{2} n_{0} / 2, \gamma_{1}>\gamma_{2}$ 
In order to have a solution we must have $\mathrm{dn} / \mathrm{dt}=\mathrm{AdT} \mathrm{T}_{2} / \mathrm{dt}$ and $\mathrm{dT}_{1} / \mathrm{dt}=\mathrm{CdT}_{2} / \mathrm{dt}$ as $\mathrm{T}_{2}$ becomes large along the line determined by the assumed equations.

$$
\begin{aligned}
\frac{d n}{d t} & =\frac{\rho_{0}-\alpha_{1} T_{1}-\alpha_{2} T_{2}}{\ell} n \\
& =\frac{\rho_{0}-\alpha_{1} C T_{2}-\alpha_{1} D-\alpha_{2} T_{2}}{\ell}\left(A T_{2}+B\right) \\
& =\frac{\rho_{0}-\alpha_{1} D}{\ell} B+\frac{\left(\rho_{0}-\alpha_{1} D\right) A-\left(\alpha_{1} C+\alpha_{2}\right) B}{\ell} T_{2}-\frac{\alpha_{1} C+\alpha_{2}}{\ell} A T_{2}^{2} \\
A \frac{d T}{d t} & =A K_{2} n-A \gamma_{2} T_{2} \\
& =A K_{2}\left(A T_{2}+B\right)-A \gamma_{2} T_{2} . \\
& =A B K_{2}+A\left(A K_{2}-\gamma_{2}\right) T_{2} .
\end{aligned}
$$

Setting the $\mathrm{T}_{2}^{2}$ term to zero we have

$$
\alpha_{1} c+\alpha_{2}=0
$$

Equating linear terms,

$$
\frac{\rho_{0}-\alpha_{1} D}{\ell}=A K_{2}-r_{2}
$$

Looking at the other condition we have

$$
\begin{aligned}
\frac{d T_{1}}{d t} & =K_{1} n-\gamma_{1} T_{1}=K_{1}\left(A T_{2}+B\right)-\gamma_{1}\left(C T_{2}+D\right) \\
& =\left(K_{1} B-\gamma_{1} D\right)+\left(A K_{1}-\gamma_{1} C\right) T_{2} \\
C \frac{d T_{2}}{d t} & =C K_{2} n-C_{\gamma_{2}} T_{2}
\end{aligned}
$$




$$
\begin{aligned}
& =\mathrm{CK}_{2}\left(\mathrm{AT}_{2}+\mathrm{B}\right)-\mathrm{C}_{2} \mathrm{~T}_{2} \\
& =\mathrm{BCK}_{2}+\mathrm{C}\left(\mathrm{AK}_{2}-{\gamma_{2}}_{2}\right) \mathrm{T}_{2} .
\end{aligned}
$$

Equating linear terms we have

$$
A K_{1}-C \gamma_{1}=C\left(A K_{2}-\gamma_{2}\right)
$$

For the two region model we obtain three equations in $A, C$, and $D$ which can be solved directly. The constant $B$ remains undetermined, thus the space contains a plane of lines which are approached asymptotically by trajectories. We note, however, that B is contained in the constant part of the above equations, thus as B becomes large the value of $\mathrm{T}_{2}$ required for the linear term to dominate becomes large.

\section{Nonlinear Analysis - Boundary Planes}

The planes formed by the equations $\mathrm{n}=\mathrm{AT}_{2}+\mathrm{B}$ and $\mathrm{T}_{1}=\mathrm{CT}_{2}+\mathrm{D}$ intersect with the $n=0$ plane to form an infinite cone-shaped region with the property that all trajectories cross the plane boundaries of the region towards the interior. To prove this we define the functions $f_{1}=A T_{2}-n_{2} f_{2}=C_{2}-T_{1}$, and we need to show that

$$
\begin{aligned}
& \nabla f_{1} \cdot \dot{X} \geqslant 0 \quad\left(n=A T_{2}+B, T_{1} \leqslant C T_{2}+D\right) \\
& \nabla f_{2} \cdot \dot{X} \geqslant 0 \quad\left(T_{1}=C T_{2}+D, 0 \leqslant n \leqslant A T_{2}+B\right)
\end{aligned}
$$

where the vector $x$ is defined by $x=\left(n_{1}, T_{1}, T_{2}\right)$. Looking first at Eq. (3-25), . . 


$$
\begin{aligned}
\nabla f_{1} \cdot \frac{d X}{d t} & =A \frac{d T_{2}}{d t}-\frac{d n}{d t} \\
& =A K_{2} n-A \gamma_{2} T_{2}-\frac{\rho_{0}-\alpha_{1} T_{1}-\alpha_{2} T_{2}}{\ell} n .
\end{aligned}
$$

If we assume $\alpha_{1} \leqslant 0, n \geqslant 0$, we have

$$
\frac{\alpha_{1} \mathrm{n}}{l} \mathrm{~T}_{1} \geqslant \frac{\alpha_{1} \mathrm{n}}{l}\left(\mathrm{CT}_{2}+\mathrm{D}\right)
$$

Inserting this and substituting for $\mathrm{n}$ we have

$$
\begin{aligned}
& \nabla f_{1} \cdot \dot{X} \geqslant A K_{2}\left(A T_{2}+B\right)-A \gamma_{2} T_{2}-\frac{\rho_{0}-\alpha_{1} C T_{2}-\alpha_{1} D-\alpha_{2} T_{2}}{\ell}\left(A T_{2}+B\right) \\
& =\left(A K_{2}-\frac{\rho_{0}-\alpha_{1} D}{\ell}\right) B+\left[A\left(A K_{2}-\gamma_{2}-\frac{\rho_{0}-\alpha_{1} D}{\ell}\right)+\frac{\alpha_{1} C+\alpha_{2}}{\ell} B\right] T_{2} \\
& +\frac{\alpha_{1} C_{1}+\alpha_{2}}{\ell} T_{2}^{2} .
\end{aligned}
$$

Using Eqs. (3-21) and (3-22) from the previous section we have

$$
\nabla f_{1} \cdot \frac{d x}{d t} \geqslant \gamma_{2}{ }^{B}
$$

Restricting $B$ to positive values we have

$$
\nabla f_{1} \cdot \dot{X} \geqslant 0 \quad\left(0 \leqslant n=A T_{2}+B, T_{1} \leqslant C T_{2}+D, \alpha_{1} \leqslant 0\right) .
$$

For the second part of the proof we have

$$
\begin{aligned}
\nabla f_{2} \cdot \dot{x} & =C d T_{2} / d t-d T_{1} / d t \\
& =C K_{2} n-C \gamma_{2} T_{2}-K_{1} n+\gamma_{1} T_{1} .
\end{aligned}
$$

If we assume $\mathrm{CK}_{2}-\mathrm{K}_{1} \leqslant 0, \mathrm{n} \geqslant 0$,

$$
\left(\mathrm{CK}_{2}-\mathrm{K}_{1}\right) \mathrm{n} \geq\left(\mathrm{CK}_{2}-\mathrm{K}_{1}\right)\left(\mathrm{AT} \mathrm{T}_{2}+\mathrm{B}\right)
$$


Inserting and substituting for $T_{1}$,

$$
\begin{aligned}
& \nabla f_{2} \cdot \dot{X} \geqslant\left(C K_{2}-K_{1}\right)\left(A T_{2}+B\right)-C_{\gamma_{2}} T_{2}+\gamma_{1}\left(C T_{2}+D\right) \\
& =B\left(C K_{2}-K_{1}\right)+\gamma_{1} D+\left[A\left(C K_{2}-K_{1}\right)+C\left(\gamma_{1}-Y_{2}\right)\right] T_{2} .
\end{aligned}
$$

Using Eq. (3-23) to eliminate the $\mathrm{T}_{2}$ term,

$$
\nabla f_{2} \cdot \dot{X} \geqslant B\left(C K K_{2}-K_{1}\right)+\gamma_{1} D
$$

If $B$ is such that $B\left(C K_{2}-K_{1}\right)+\gamma_{1} D \geqslant 0$, we have $\nabla f_{2} \cdot \dot{X} \geqslant 0$ for $0 \leqslant n \leqslant A T_{2}+B, T_{2}=C T_{1}+D, C K_{2}-K_{1} \leqslant 0$. Noting that the largest value of $B$ for which the proof holds will result in the largest region, we obtain an equation for $B$.

$$
B=\frac{Y_{1} D}{K_{1}-C K_{2}} \text {. }
$$

From the assumptions involved in the proof we can obtain the region of the parameter space for which the proof is valid. Using the variables defined in the linear analysis, the assumptions $\alpha_{1} \leq 0$, $\mathrm{CK}_{2}-\mathrm{K}_{1} \leqslant 0$ can be written as

$$
x+y \leq 0
$$

In restricting the analysis to physically meaningful parameters, we must have $n_{0} \geqslant 0$ for $\rho_{0} \geqslant 0$ which gives us

$$
\gamma_{2} x+\gamma_{1} y \geq 0
$$

Another implicit assumption in the second part of the proof is that $D \geqslant 0$, solving the equations for $D$ we have 


$$
D=\frac{1}{\alpha_{1}}\left(\rho_{0}+\frac{\alpha_{1} K_{1} \gamma_{2}+\alpha_{2} K_{2} \gamma_{1}}{\ell}\right) .
$$

Performing some algebra and converting to the parameter space variables, we have

$$
x+y \geqslant-\gamma_{1} \gamma_{2}
$$

All of the inequalities are satisfied in the shaded region, BGOC, of Fig. 6.

It should be noted that since the region defined here is open to Infinity, we have not proven boundedness for trajectories entering into or originating within the region. Application of Liapunov techniques to the problem were unsuccessful, as were attempts at a geometric proof of boundedness. However, in numerlcal trajectory calculations, no unstable trajectories could be found which originated within the region. Computer calculations of the stability boundary for one of the cases showed the region found here to be contained within the actual stable region. In addition, parameter conditions corresponding to the disappearance of stable limit cycles given in a previous paper (Shotkin, 1964) coincide with Eq. (3-32). Assuming the region found here to contain only bounded solutions, we can consider region $B G O C$ of the parameter space to be $a^{\circ}$ region of conditional nonlinear stability in that the equilibrium power can be increased to a point where the stable limit cycle disappears and the entire state space becomes unstable.

\section{Numerical Example - Decoupled System}

In selecting parameter values for the two region model we note that the equations can be reduced to the model studied in Chapter 2. 
If we let $\gamma_{1}$ become infinite while $\gamma_{1} / K_{1}$ remains finite and neglect $d T_{1} / d t$ we have

$$
\begin{aligned}
& \frac{d n}{d t}=\frac{\rho_{0}-\alpha_{1} K_{1} n / \gamma_{1}-\alpha_{2} T_{2}}{\ell} \\
& \frac{d T_{2}}{d t}=K_{2} n-\gamma_{2} T_{2} .
\end{aligned}
$$

This corresponds to assuming zero heat capacity in region 1 . For the numerical example the parameter values used are $\rho_{0} / \ell=6, \alpha_{1} / \ell=-1$, $K_{1}=1000, \gamma_{1}=1000, \alpha_{2} / l=1, K_{2}=50, \gamma_{2}=10$. Note that if we let $K_{1}=r_{1}+\infty$ this example would reduce to the example problem of Chapter 2. Using these parameters the coefficients defining the boundary planes are $A=1.042, B=38.005, C=1.000, D=36.105$. The boundary planes for this case are shown in Fig. 7 along with system trajectories calculated for a set of initial conditions obtained by perturbing power from the operating equilibrium point. That part of the trajectories not contained in the derived region is shown in dashed lines. Using the same parameters two trafectories were calculated for initial conditions near the separatrix surface for the system. The projection of these trajectories on the $T_{2}=0, T_{1}=0$, and $n=0$ planes is shown in Figs. 8, 9, and 10. The dashed line in these figures is the projected intersection of the two planes.

\section{Numerical Example - Coupled System}

As shown in a previous paper (Schmidt, 1969), Eqs. (3-5) and (3-6) for the coupled system can be converted to the decoupled form by making the transformation $T_{1}^{c}=T_{1}^{d}, T_{2}^{c}=T_{2}^{d}-n_{2} T_{1}^{d} /\left(n_{1}-n_{3}\right)$ 


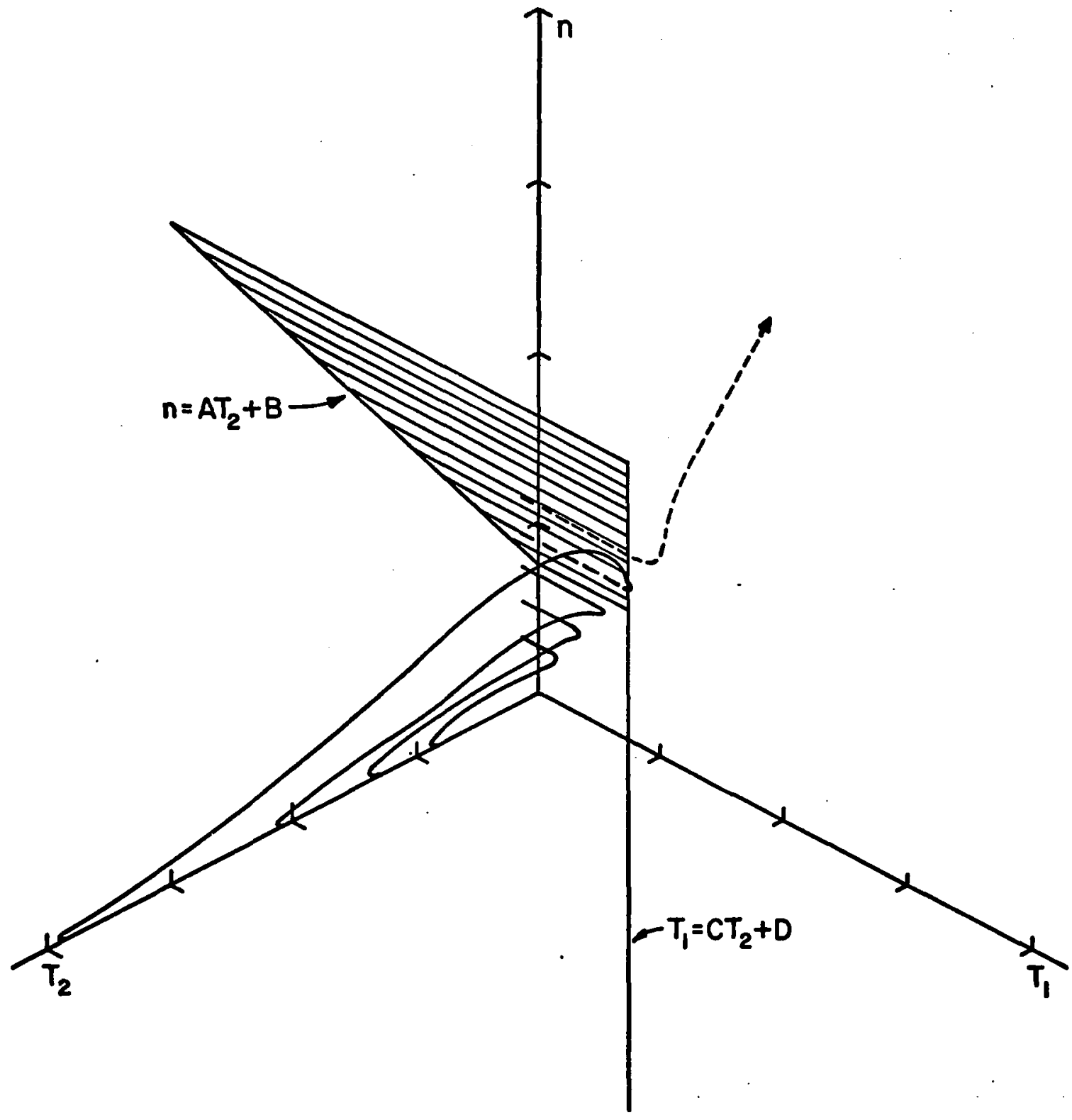

Fig. 7. System Trajectories and Boundary Rlanes, Two Tempęature Region Model, Decoupled Systè 


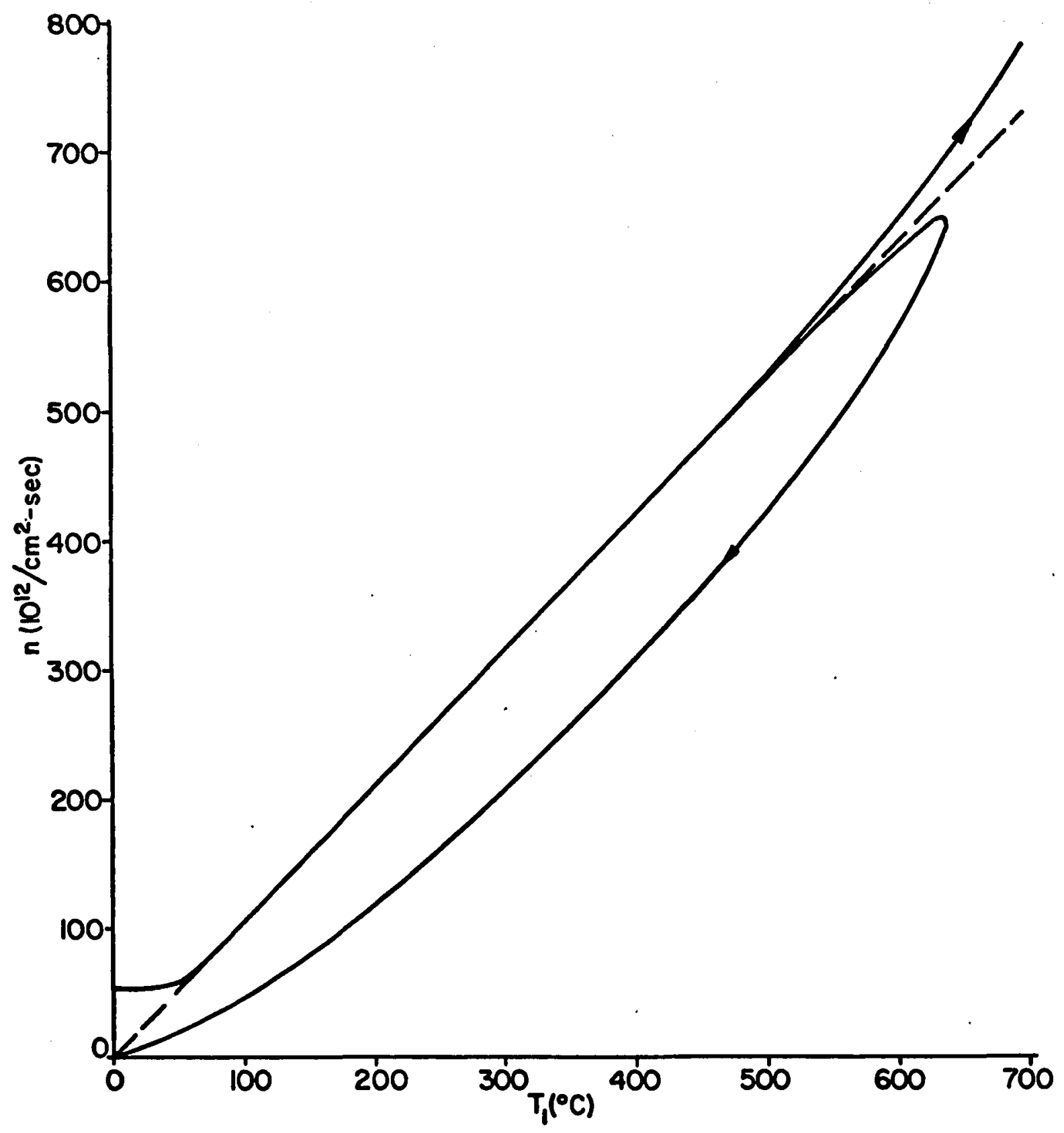

F18. 8. Projection of System Trajectories on $n, T$ Plane, Two Temperature Region Mode1, Decoupled System 


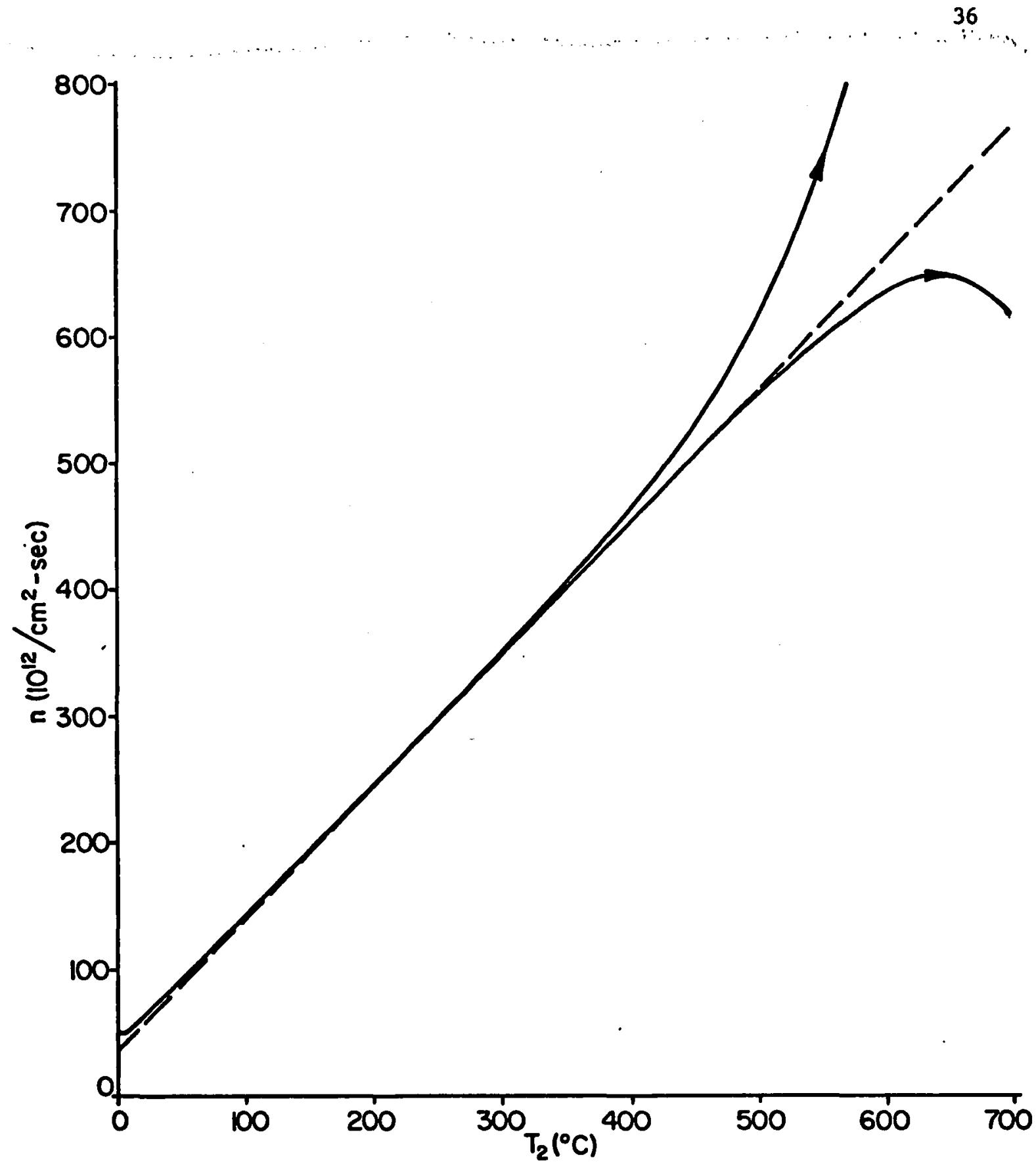

F18. 9. Projection of System Trajectories on $n, r_{2}$ Plane, Two Temperature Region Model, Decoupled System 


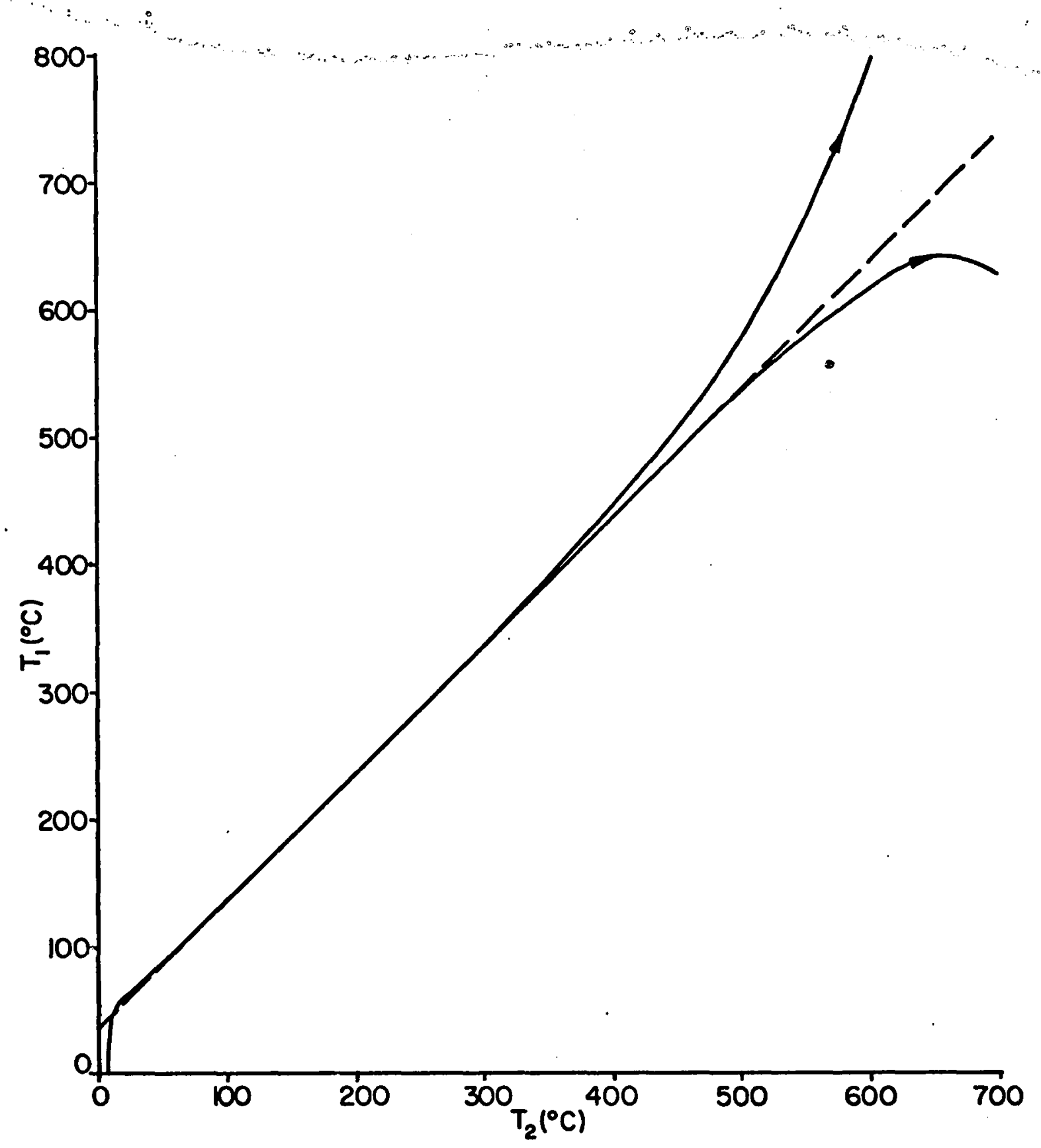

F18. 10. Projéction of System Trajectories on $T_{1}, T_{2}$ Pianes. Two Temperature Region Model, Decoupled System 
where $c$ and $d$ refer to the coupled and decoupled systems. Since the two system formulations are equivalent, the results of this chapter can be applied to the coupled system by transforming the region boundaries obtained for the equivalent decoupled system into the coupled system state space.

The parameter values for this example are taken from a problem studied by Schmidt. Although the numbers obtained from the analysis become rather astronomical, the results are applicable as a study of the mathematical model. The parameter values for this case are $\rho_{0} / \ell=0.08833, \alpha_{1} / l=-0.333 \times 10^{-5}, \alpha_{2} / \ell=0.4 \times 10^{-5}, \eta_{1}=0.5, \eta_{2}=0.25$, $n_{3}=0.2, b=100$. The boundary plane equations are

$$
\begin{aligned}
\mathrm{n} & =\left(3.0 \times 10^{-3}\right) \mathrm{T}_{1}+\left(3.6 \times 10^{-3}\right) \mathrm{T}_{2}+17.5 \\
\mathrm{~T}_{1} & =1.2 \mathrm{~T}_{2}+3.5 \times 10^{3}
\end{aligned}
$$

Figure 11 shows a portion of the boundary planes contained in the region $\mathrm{n}>0, \mathrm{~T}_{1}>0, \mathrm{~T}_{2}>0$. To determine the relationship of the actual separatrix surface to the surface found here, the actual surface was determined by computer for a set of values of $T_{1}$. The intersection of the stability boundary with the $T_{1}=$ constant plane is shown in Fig. 12 for several values of $T_{1}$. The origin in each case is the intersection of the planes defined by Eqs. (3-35) and (3-36). The region bounded by these planes is contained within the stability boundary fi: the system, and as $T_{1}$ becomes large the boundary approaches the constant reactivity plane defined by Eq. (3-36). 


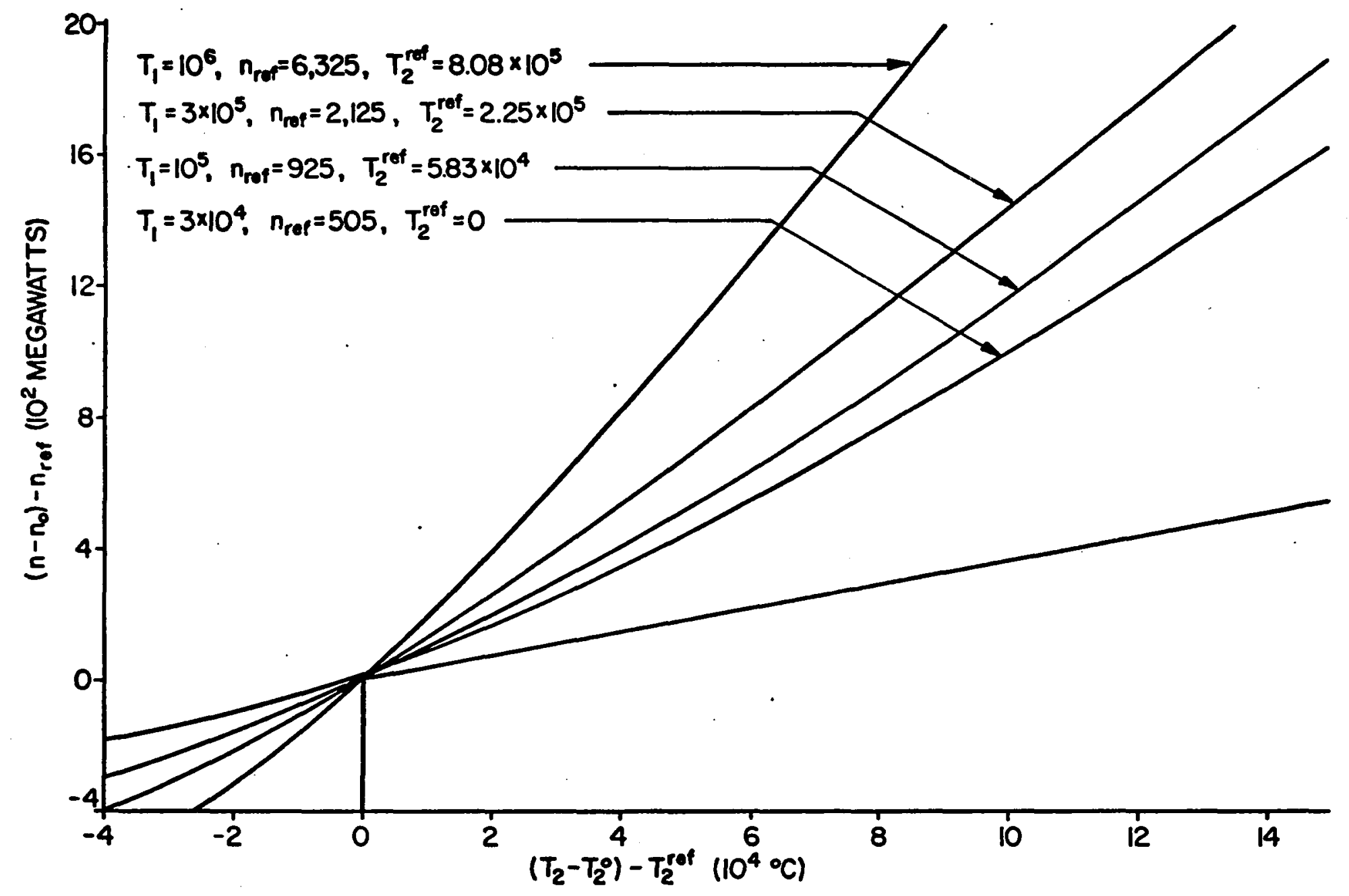

Fig. 12. Comparison of Boundary Planes to Actual Stab1lity Boundary 
CHAPTER 4

APPLICATION TO HIGHER ORDER SYSTEMS

Delayed neutron effects were neglected in the mathematical models studied in the previous chapters to simplify the analysis. The effect of adding delayed neutrons to the model is to increase the dimension of the phase space by one dimension for each delayed neutron group included. Since the method used here is highly geometric, interpretation of results becomes more difficult for systems of fourth order or higher. The simplest system containing delayed neutrons which exhibits conditional nonlinear stability is obtained by adding one delayed neutron group to the model studied in Chapter 2.

One Delay Group, One Region, Power Coefficient

The differential equations for this system are

$$
\begin{aligned}
& \frac{d \mathfrak{n}}{d t}=\frac{\rho_{0}-\beta-\alpha T-k \mathfrak{n}}{\ell} \mathbf{n}+\lambda c \\
& \frac{d c}{d t}=\frac{\beta}{\ell} \mathbf{n}-\lambda c \\
& \frac{d T}{d t}=k n-\gamma T
\end{aligned}
$$

Following the approach used for the previous systems, we assume a set of solution trajectories approach the form $n=A T+B, c=D T+E$, for T large. Substituting these equations into the system differential equations and solving for the coefficients for which $\mathrm{dn} / \mathrm{dt}=\mathrm{AdT} / \mathrm{dt}$ and 
$\mathrm{dc} / \mathrm{dt}=\mathrm{DdT} / \mathrm{dt}$ for $\mathrm{T}$ large we have

$$
\begin{aligned}
& A=-\frac{\alpha}{\kappa} \\
& B=\frac{1}{K}\left(\rho_{0}-\omega_{p} \ell-\frac{\hat{\beta} \omega_{p}}{\omega_{p}+\lambda}\right) \\
& D=\frac{-\alpha \beta}{K \ell\left(\omega_{p}+\lambda\right)}
\end{aligned}
$$

where

$$
\omega_{p}=-\left(\gamma+\frac{\alpha K}{k}\right)
$$

As in the system studied in Chapter 3 we see that one of the coefficients remains arbitrary, indicating the set of asymptotically linear trajectories approaches a plane perpendicular to the $n, T$ plane. From the value of $A$ we note that the equation for this plane has the form $\kappa n+\alpha T=$ constant, thus as in the two previous cases the asymptotically linear trajectories approach lines of constant reactivity. In solving for the value of the reactivity in the plane we have

$$
\rho_{p}-\omega_{p} \ell-\frac{\beta \omega_{p}}{\omega_{p}+\lambda}=0 .
$$

Note that this equation has the same form as the inhour equation; if the derived line were a trajectory, the inverse period along the line would be $\omega_{p}$.

For a numerical example of this system we add delayed neutron parameters to the system studied in Chapter 2. The parameters. used are $\rho_{0} / \ell=6, \alpha / \ell=1, k / \ell=-1, \beta / \ell=100, \lambda=0.1, k=50, \gamma=10$. For this case the constants defining the asymptote lines are $A=1, B=133.75, D=2.494$. 
The dashed line of Fig. 13 is the projection of these lines on the n, T plane. The trajectories shown were calculated for initial conditions obtained by perturbing power from the equilibrium point to points near the actual separatrix surface of the system. These and other trajectories calculated for initial conditions near the separatrix surface indicate the actual stability boundary approaches the defined constant reactivity plane as $\mathrm{T}$ becomes large.

\section{Higher Order Systems}

In going to higher order mathematical models, differential equations of the form $d c_{i} / d t=\left(\beta_{i} / \ell\right) n-\lambda_{i} c_{i}$ or $d T_{i} / d t=K_{i} n-\gamma_{i} T_{i}$ are added to the system. In studying the effect of adding equations of this form to the system we see that for each additional dimension, two additional coefficients are needed to determine uniquely a line in the space, however in solving for these coefficients we obtain only one linear equation per added dimension. Thus the dimension of the subspace containing asymptote lines is always one less than the dimension of the system. The only second order term is in the $\mathrm{dn} / \mathrm{dt}$ equation, and is such that setting this term to zero results in a constant reactivity condition. Thus the subspace discussed above is one of constant reactivity. 


$$
b
$$




\section{CHAPTER 5}

\section{SUMMARY}

The point reactor kinetics model with linear reactivity feedback contains a set of trajectories which approach infinity along lines of constant reactivity. By solving for the set of asymptotes, we can gain information which is helpful in defining regions of system stability.

Applying this technique to the effective lifetime model with one temperature region and a power coefficient, a variable transformation was found which simplifies the analysis. Using this transformation, an exact solution was obtained for the case where the system is a nonlinear center. In addition, a Liapunov function was developed which proves asymptotic stability for a region of the state space which is much larger than that shown by previous methods.

The results for the effective lifetime model with two temperature regions were less conclusive, but the technique did provide new information about the system. For this case the set of asymptotes are contained in a plane of constant reactivity which appears to be approached by the actual boundary of the stable region for $T$ large in the numerical cases studied. In addition, a region bounded by three intersecting planes was found which was contained within the stable region demonstrated by computer solutions of the numerical examples. 
Although interpretation of results becomes more difficult in higher order models, the set of asymptotes can be found by a straightforward application of the method used on the simpler models. The dimension of the subspace containing these asymptotes is always one less than that of the state space. Further study of the models indicates this subspace is one of constant reactivity.

In each of the models studied, a subspace containing trajectories which approach infinity with constant reactivity was shown to exist. Investigation of numerical examples indicate this subspace contains a very special class of trajectories in that unbounded trajectories not in the subspace approach infinity with increasing reactivity. If this is true, further speculation leads to the possibility that this subspace forms a boundary between stable and unstable regions of the state space. In further study of these possibilities it could be helpful to use techniques developed for describing behavior of the system at infinity (Sinets, 1970) 


\section{REFERENCES}

Devooght, J:, and H. B. Smets, "Determination of Stability Domains in Point Reactor Dynamics," Nuc1. Sci. and Eng., 28, 226-236 (1967).

Hetrick, David L., Dynamics of Nuclear Reactors, University of Chicago Press, Chicago (1971).

Schmidt, Theodore R., "Nonlinear Oscillations and Stability of a Nuclear Reactor with Two Reactivity Feedbacks," Ph.D. Dissertation, University of Arizona (1969).

Schultz, D. G., "The Generation of Liapunov Functions," Advances in Control Systems, vo1. 2, 1-64, Academic Press, Inc., New York (1965).

Shotkın, Louis M., "A Nonlinear Analysis of a Reactor with Two Temperature Coefficients," Nucl. Sci. and Eng., 18, 271-279 (1964).

Shotkin, Louis M., "Instability Bounds in Linearly Stable Systems," Nucl. Sci. and Eng., 35, 211-219 (1969).

Smets, Henri B., "Unlimited Power Excursions in Nuclear Reactors," paper presented at the Conference on Dynamics of Nuclear Systems, University of Arizona, Tucson Arizona, March 23-25, 2970 (proceedings to be published by University of Arizona Press). 